\title{
THE SPLITTING OF COHOMOLOGY OF $p$-GROUPS WITH RANK 2
}

\author{
AKIHIKO HIDA AND NOBUAKI YAGITA
}

\begin{abstract}
Let $p$ be an odd prime and $B P$ the classifying space of a $p$-group $P$ with $\operatorname{rank}_{p}(P)=2$. By using stable homotopy splitting of $B P$, we study the decomposition of $H^{\text {even }}(P ; \mathbb{Z}) / p$ and $C H^{*}(B P) / p$.
\end{abstract}

\section{INTRODUCTION}

Let $P$ be a $p$-group and $B P$ its classifying space. We study the stable splitting and splitting of cohomology

$$
\begin{gathered}
(*) \quad B P \cong X_{1} \vee \ldots \vee X_{i}, \\
(* *) \quad H^{*}(P) \cong H^{*}\left(X_{1}\right) \oplus \ldots \oplus H^{*}\left(X_{i}\right) \quad(\text { for } *>0)
\end{gathered}
$$

where $X_{i}$ are irreducible spaces in the stable homotopy category. If we can get the splitting $(* *)$ of cohomology, then we can study $H^{*}(G)_{(p)}$ for all groups $G$ having Sylow $p$ subgroups which are isomorphic to $P$.

When Mitchell and Priddy [Mi], [Mi-Pr] started this problem, the splitting $(*)$ was got by using the splitting $(* *)$ of the cohomology of $P$. Since $H^{*}(P)$ are quite complicated for odd primes nonabelian $p$-groups, the examples were mostly given for $p=2$.

But after the answer of the Segal conjecture by Carlsson, the splittings $(*)$ are given by only using modular representation theory by Nishida [Ni], Benson-Feshbach [Be-Fe] and Martino-Priddy [Ma-Pr]. In fact, their theorems say that such decomposition is decided only by structures of simple modules of the $\bmod (p)$ double Burnside algebra $A(P, P)$. These theorems do not use splittings of cohomology, and results are given for all primes $p$.

In particular, Dietz and Dietz-Priddy [Di], [Di-Pr] gave the stable splitting $(*)$ for groups $P$ with $\operatorname{rank}_{p}(P)=2$ for $p \geq 5$. However it was not used splittings $(* *)$ of the cohomology $H^{*}(P)$, and the cohomologies $H^{*}\left(X_{i}\right)$ were not given there.

2010 Mathematics Subject Classification. Primary 55P35, 57T25, 20C20 ; Seconary 55R35, 57T05.

Key words and phrases. stable splitting, cohomology, classifying spaces. 
In $\left[\right.$ Hi-Ya1,2], we give the cohomology of $H^{*}\left(X_{i}\right)$ (and hence $(* *)$ ) for $P=p_{+}^{1+2}$ the extraspecial $p$ group of order $p^{3}$ and exponent $p$. Their cohomology $H^{*}\left(X_{i}\right)$ are very complicated but have rich structures, in fact $p_{+}^{1+2}$ is a $p$-Sylow subgroup of many interesting groups, e.g., $G L_{3}\left(\mathbb{F}_{p}\right)$ and many simple groups e.g. $J_{4}$ for $p=3$.

In this paper, we give the decomposition of

$$
H^{*}(P)=H^{*}(P ; \mathbb{Z}) /(p, \sqrt{0}) \quad\left(\text { and } \quad H^{e v}(P)=H^{\text {even }}(P ; \mathbb{Z}) / p\right)
$$

for other $\operatorname{rank}_{p} P=2$ groups for odd primes $p$. It is important to compute the transfer map for the double Burnside algebra $A(P, P)$ action on $H^{*}(P ; \mathbb{Z})$. In general, it is not a so easy problem to compute the transfer on $H^{*}(P ; \mathbb{Z})$. However we can always compute it on $H^{*}(P)$ from Quillen's theorem.

In most cases, $H^{*}\left(X_{i}\right)$ are easily got but seemed not to have so rich structure as $p_{+}^{1+2}$, because they are not $p$-Sylow subgroups of so interesting groups. However, we hope that from our computations, it becomes more clear the relations among splittings of $H^{*}(P)$ of groups $P$ with $\operatorname{rank}_{p}(P)=2$.

In particular, we note that the irreducible components are most fine in those of rank $_{p}=2$ groups, namely, the cohomology $H^{*}\left(X_{i}(P)\right)$ can be written by using the decomposition $H^{*}\left(X_{k}\left(p_{+}^{1+2}\right)\right.$ ) (Lemma 8.1, Theorem 8.2, Theorem 8.5).

Theorem 1.1. For $p \geq 5$, let $P$ be a non-abelian $p$-group of $\operatorname{rank}_{p} P=$ 2 , which is not a metacyclic group. Let $X_{i}(P)$ be an irreducible component of BP. Then we can write

$$
H^{*}\left(X_{i}(P)\right) \cong \oplus_{j \in J(i, P)} H^{*}\left(X_{j}\left(p_{+}^{1+2}\right)\right)
$$

for some index set $J(i, P)$.

This paper is organized as follows. In $\S 2$ we recall the definition and properties of the double Burnside algebra and the stable splitting. In $\S 3$, we note the relations between splittings when cohomology of groups are isomorphic. In $\S 4-\S 7$, we give the stable splitting of the cohomology of $H^{*}(P)$ for the elementary abelian group $\mathbb{Z} / p \times \mathbb{Z} / p$, metacyclic groups, $C(r)$ groups (such that $\left.C(3)=p_{+}^{1+2}\right)$, and $G\left(r^{\prime}, e\right)$ groups which appeared in the classification of $\operatorname{rank}_{p}(P)=2$ groups for $p \geq 5$ respectively. However, some parts in $\S 4, \S 6$ are still given in [Hi-Ya1]. In $\S 8$, we study the relation among groups studied in $\S 5-\S 7$. In $\S 9$ we study the nilpotent ideal parts in $H^{e v}(P)=H^{e v e n}(P ; \mathbb{Z}) / p$ for all groups in $\S 4-\S 7$. In $\S 10$, we note the relation to the Chow ring $C H^{*}(B P) / p$ and $H^{e v}(P)$, and note that the Chow group of the direct 
summand $X_{i}$ is represented by some motive of the classifying space $B P$.

\section{The DOUble Burnside Algebra AND STABle SPlitTing}

Let us fix an odd prime $p$ and $k=\mathbb{F}_{p}$. For finite groups $G_{1}, G_{2}$, let $A_{\mathbb{Z}}\left(G_{1}, G_{2}\right)$ be the double Burnside group defined by the Grothendieck group generated by $\left(G_{1}, G_{2}\right)$-bisets. Each element $\Phi$ in $A_{\mathbb{Z}}\left(G_{1}, G_{2}\right)$ is generated by elements $[Q, \phi]=\left(G_{1} \times{ }_{(Q, \phi)} G_{2}\right)$ for some subgroup $Q \leq G_{1}$ and a homomorphism $\phi: Q \rightarrow G_{2}$. In this paper, we use the notation

$$
[Q, \phi]=\Phi: G_{1} \geq Q \stackrel{\phi}{\rightarrow} G_{2} .
$$

For each element $\Phi=[Q, \phi] \in A_{\mathbb{Z}}\left(G_{1}, G_{2}\right)$, we can define a map from $H^{*}\left(G_{2} ; k\right)$ to $H^{*}\left(G_{1} ; k\right)$ by

$$
x \cdot \Phi=x \cdot[Q, \phi]=\operatorname{Tr}_{Q}^{G_{1}} \phi^{*}(x) \quad \text { for } x \in H^{*}\left(G_{2} ; k\right) .
$$

When $G_{1}=G_{2}$, the group $A_{\mathbb{Z}}\left(G_{1}, G_{2}\right)$ has the natural ring structure, and call it the (integral) double Burnside algebra. In particular, for a finite group $G$, we have an $A_{\mathbb{Z}}(G, G)$-module structure on $H^{*}(G ; k)$ (and $H^{*}(G ; \mathbb{Z}) / p$ ).

Recall Quillen's theorem such that the restriction map

$$
H^{*}(G ; k) \rightarrow \lim _{V} H^{*}(V ; k)
$$

is an F-isomorphism (i.e. the kernel and cokernel are nilpotent) where $V$ ranges elementary abelian $p$-subgroups of $G$. We easily see ([HiYa1])

Lemma 2.1. Let $\sqrt{0}$ be the nilpotent ideal in $H^{*}(G ; k)\left(\right.$ or $\left.H^{*}(G ; \mathbb{Z}) / p\right)$. Then $\sqrt{0}$ itself is an $A_{\mathbb{Z}}(G, G)$-module.

In this paper we first (in $\S 4-\S 8$ ) consider the cohomology modulo nilpotent elements, since it is not so complicated from the above Quillen's theorem. Hence we write it simply

$$
H^{*}(G)=H^{*}(G ; \mathbb{Z}) /(p, \sqrt{0}) .
$$

However we also compute $H^{\text {even }}(G ; \mathbb{Z}) / p$ in $\S 9$ below.

By the preceding lemma, we see that $H^{*}(G)$ has the $A_{\mathbb{Z}}(G, G)$ module structure. (Here note that $A_{\mathbb{Z}}(G, G)$ acts on unstable cohomology.) For ease of notations and arguments, when there is an $A_{\mathbb{Z}}(G, G)$-filtration $F_{1} \subset \ldots \subset F_{n} \cong H^{*}(P)$ such that

$$
\operatorname{gr} H^{*}(P)=\oplus F_{i+1} / F_{i} \cong \oplus m_{i} M_{i} \quad \text { for } *>0
$$


with simple $A_{\mathbb{Z}}(G, G)$-modules $M_{i}$, we simply write

$$
H^{*}(G) \leftrightarrow \oplus m_{i} M_{i}
$$

Throughout this paper, we assume that degree $*>0$ (or we consider $H^{*}(-)$ as the reduced theory $\tilde{H}^{*}(-)$ ). (We consider $H^{*}(G)$ as an element in $K_{0}\left(\operatorname{Mod}\left(A_{\mathbb{Z}}(G, G)\right)\right)$.) In this paper, $H^{*}(G) \cong A$ for an graded ring $A$ means an graded module isomorphism otherwise stated, while (in most cases) it is induced from the ring isomorphism $\operatorname{gr} H^{*}(G) \cong A$ for some filtrations of $H^{*}(G)$.

Let $B G=B G_{p}$ be the $p$-completion of the classifying space of $G$. Recall that $\{B G, B G\}_{p}$ is the ( $p$-completed) group generated by stable homotopy self maps. It is well known from the Segal conjecture (Carlsson's theorem) that this group is isomorphic to the double Burnside group $A_{\mathbb{Z}}\left(G_{1}, G_{2}\right)^{\wedge}$ completed by the augmentation ideal.

Since the transfer is represented as a stable homotopy map $\mathrm{Tr}$, an element $\Phi=[Q, \phi] \in A\left(G_{1}, G_{2}\right)$ is represented as a map $\Phi \in$ $\left\{B G_{1}, B G_{2}\right\}_{p}$

$$
\Phi: B G_{1} \stackrel{T r}{\rightarrow} B Q \stackrel{B \phi}{\rightarrow} B G_{2} .
$$

(Of course, the action for $x \in H^{*}\left(G_{2}\right)$ is given by $\operatorname{Tr}_{Q}^{G_{1}} \phi^{*}(x)$ as stated.)

Let us write

$$
A\left(G_{1}, G_{2}\right)=A_{\mathbb{Z}}\left(G_{1}, G_{2}\right) \otimes k \quad(k=\mathbb{Z} / p) .
$$

Hereafter we consider the cases $G_{i}=P ; p$-groups. Given a primitive idempotents decomposition of the unity of $A(P, P)$

$$
1=e_{1}+\ldots+e_{n}
$$

we have an indecomposable stable splitting

$$
B P \cong X_{1} \vee \ldots \vee X_{n} \text { with } e_{i} B P=X_{i} .
$$

In this paper, an isomorphism $X \cong Y$ for spaces means that it is a stable homotopy equivalence.

Recall that

$$
M_{i}=A(P, P) e_{i} /\left(\operatorname{rad}\left(A(P, P) e_{i}\right)\right.
$$

is a simple $A(P, P)$-module where $\operatorname{rad}(-)$ is the Jacobson radical. By Wedderburn's theorem, the above decomposition is also written as

$$
B P \cong \vee_{j}\left(\vee_{k} X_{j k}\right)=\vee_{j} m_{j} X_{j 1} \text { where } m_{j}=\operatorname{dim}\left(M_{j}\right)
$$

for $A(P, P) e_{j k} / \operatorname{rad}\left(A(P, P) e_{j k}\right) \cong M_{j}$. Therefore the stable splitting of $B P$ is completely determined by the idempotent decomposition of the unity in the double Burnside algebra $A(P, P)$. 
For a simple $A(P, P)$-module $M$, define a stable summand $X(M)$ by

$$
e_{M}=\sum_{M_{i} \cong M} e_{i}, \quad X(M)=\vee_{M_{j k} \cong M} X_{j k}=e_{M} B P .
$$

Here $X(M)$ is only defined in the stable homotopy category. (So strictly, the cohomology ring $H^{*}(X(M))$ is not defined.) However we can define $H^{*}(X(M))$ as a graded submodule of the cohomology ring $H^{*}(P)$ by

$$
H^{*}(X(M))=H^{*}(P) \cdot e_{M} \quad\left(=e_{M}^{*} H^{*}(P) \text { stabely }\right)
$$

where we think $e_{M} \in A(P, P)$ (rather than $e_{M} \in\{B P, B P\}$ ).

Lemma 2.2. Given a simple $A(P, P)$-module $M$, the cohomology $\operatorname{grH}^{*}(X(M))$ is isomorphic to a sum of $M$, i.e., (for $\left.*>0\right)$

$$
H^{*}(X(M)) \leftrightarrow \oplus_{i=1} M\left[k_{i}\right], \quad 0 \leq k_{1} \leq \ldots \leq k_{s} \leq \ldots
$$

where $\left[k_{s}\right]$ is the operation ascending degree $k_{s}$.

Proof. Let $M^{\prime}$ be a simple $A(P, P)$-module such that $M^{\prime} \not M$. Then

$$
e_{M^{\prime}} X\left(M_{i}\right)=e_{M^{\prime}} e_{M} B P=p t .
$$

Hence $e_{M^{\prime}}: H^{*}(P) \rightarrow H^{*}(P)$ restricts $e_{M^{\prime}} \mid H^{*}(X(M))=0$ (we assumed $*>0)$. This means that $H^{*}(X(M))$ does not contain $M^{\prime}$ as a summand.

From Benson-Feshbach [Be-Fe] and Martino-Priddy [Ma-Pr], it is known that each simple $A(P, P)$-module is written as

$$
S(P, Q, V) \text { for } Q \leq P \text {, and } V \text { : simple } k[\text { Out }(Q)] \text { - module. }
$$

(In fact $S(P, Q, V)$ is simple or zero.) Moreover, we have (see [Be-Fe]) an isomorphism

$$
S(P, Q, V) \cong V \cdot A(P, Q) / J(Q, V)
$$

for some $A(P, P)$-submodule $J(Q, V)$ of $V \cdot A(P, Q)$.

Thus we have the main theorem of stable splitting of $B P$.

Theorem 2.3. (Benson-Feshbach [Be-Fe], Martino-Priddy [Ma-Pr]) There are indecomposable stable spaces $X_{S(P, Q, V)}$ for $S(P, Q, V) \neq 0$ such that

$$
B P \cong \vee X(S(P, Q, V)) \cong \vee(\operatorname{dim} S(P, Q, V)) X_{S(P, Q, V)} .
$$

The direct summands $X_{S(P, P, V)}$ are called dominant summands ([Ni], [Ma-Pr]). Let $X_{S=S(P, Q, V)}$ be a non-dominant summand for a proper subgroup $Q$. Then it is known $([\mathrm{Ni}],[\mathrm{Ma}-\mathrm{Pr}])$ that the corresponding 
idempotent $e_{S} \in A(P, P)$ is generated by elements $P>Q \stackrel{\phi}{\rightarrow} P$ and $P \rightarrow Q \rightarrow P$. Hence when there is no non-trivial map $P \rightarrow Q$, we see

$$
H^{*}\left(X_{S}\right) \cong e_{S} H^{*}(B P) \subset \operatorname{Tr}_{Q}^{P} H^{*}(Q),
$$

that is, $x \in H^{*}\left(X_{S}\right)$ if and only if $\sum \operatorname{Tr}_{Q}^{P} \phi^{*}(x)=x$.

\section{RELATION AMONG GROUPS $P$}

Let $R$ be a subring of $A(P, P)$. For a simple $R$-module $S_{R}$, we can define the idempotent $e_{S_{R}}$ and the stable space $Y_{S_{R}}=e_{S_{R}} B P$ which decomposes $B P$, while it is (in general) not irreducible. In particular, we take the group algebra of the outer automorphism group Out $(P)$ as the ring $R$.

Lemma 3.1. For each Out $(P)$-simple module $S_{R_{i}}$ with dimension $n_{i}$, let us write by

$$
B P=n_{1} Y_{1} \vee \ldots \vee n_{s} Y_{s} \text { where } Y_{i}=e_{S_{R_{i}}} B P
$$

the decomposition for idempotents in Out $(P)$. Then each $Y_{i}$ decomposes

$$
Y_{i}=X_{i_{1}} \vee \ldots \vee X_{i_{m}} \quad \text { for } X_{i j}=e_{S_{i j}} B P
$$

where $e_{S_{i j}}$ are idempotents in $A(P, P)$.

When $P, P^{\prime}$ are different $p$ groups, the stable homotopy types of $B P, B P^{\prime}$ are different [Ni]. However there are many cases with $H^{*}(P) \cong$ $H^{*}\left(P^{\prime}\right)$ (However note that it seems not so often that $H^{*}(P ; \mathbb{Z}) \cong$ $H^{*}\left(P^{\prime} ; \mathbb{Z}\right)$ even if we do not assume the map $P \rightarrow P^{\prime}$ which induces the isomorphism on cohomology.) The following corollary is immediate from the above lemma.

Corollary 3.2. Let $P, P^{\prime}$ are p-groups with $i_{H}: H^{*}(P) \cong H^{*}\left(P^{\prime}\right)$. Assume that there is a ring map $i_{A}: A(P, P) \rightarrow A\left(P^{\prime}, P^{\prime}\right)$ such that $i_{H}(\Phi(x))=i_{A}(\Phi) i_{H}(x)$ for all $\Phi \in A(P, P)$ and $x \in H^{*}(P)$. Then for each splitting summand $X_{i}$ in $B P$, there are splitting summands $X_{i j}^{\prime}$ of $B P^{\prime}$ such that

$$
i_{H}^{*}\left(X_{i}\right)=H^{*}\left(X_{i_{1}}^{\prime}\right) \oplus \ldots \oplus H^{*}\left(X_{i_{s}}^{\prime}\right) .
$$

Proof. We get the result from

$$
i_{H} H^{*}\left(X_{i}\right)=i_{H}\left(e_{i} H^{*}(P)\right)=i_{A}\left(e_{i}\right) i_{H} H^{*}(P)=i_{A}\left(e_{i}\right) H^{*}\left(P^{\prime}\right) .
$$


Proposition 3.3. Let $f: B P \rightarrow B G$ be a map such that $f^{*}: H^{*}(G) \rightarrow$ $H^{*}(P)$ is injective. Let $B P=\vee X_{i}(P)$ and $B G=\vee X_{j}(G)$ be the irreducible decompositions. For each $X_{j}(G)$, there are $i_{1}, . ., i_{s}$ such that

$$
H^{*}\left(X_{j}(G)\right) \cong f^{*} H^{*}(G) \cap\left(H^{*}\left(X_{i_{1}}(P) \vee \ldots \vee X_{i_{s}}(P)\right)\right.
$$

Proof. We note each $f\left(X_{i}(P)\right)$ is contained some $X_{j}(G)$ otherwise $\left.X_{i}(P)\right)$ should be decomposed. Let $f\left(X_{i_{k}}(P)\right) \subset X_{j}(G)$. Then we have a map

$$
f^{*}: H^{*}\left(X_{j}(G)\right) \rightarrow H^{*}\left(X_{i_{1}}(P) \vee \ldots \vee X_{i_{s}}(P)\right) .
$$

Since $f^{*}$ is injective, we have the proposition.

If $G$ has Sylow $p$-group isomorphic to $P$, then of course the above proposition holds. Moreover we consider the cases that $P \subset G$ and $G$ is also a $p$-group satisfying the above proposition in $\S 5$ below.

\section{4. $A=\mathbb{Z} / p \times \mathbb{Z} / p$ FOR $p \geq 3$}

In this section, we recall the decomposition of the cohomology of $\mathbb{Z} / p \times \mathbb{Z} / p$, which is still given $\S 5$ in [Hi-Ya1]. However the result is not so trivial, and we write it briefly. (The results used in the other sections are only Lemma 4.1 and Theorem 4.4.)

We recall the cohomology

$$
H^{*}(A) \cong k[u, y], \quad \text { for } A=\langle a, b\rangle \cong \mathbb{Z} / p \times \mathbb{Z} / p,
$$

where $u=c_{1}\left(e_{a}\right)$ is the first Chern class of a non zero linear representation $e_{a}: A \rightarrow\langle a\rangle \rightarrow \mathbb{C}^{\times}$and $y=c_{1}\left(e_{b}\right)$ is defined similarly.

At first we consider the case $B=\langle b\rangle \cong \mathbb{Z} / p$ with $H^{*}(B) \cong k[y]$. The outer automorphism $\operatorname{Out}(B) \cong \mathbb{F}_{p}^{*}$ and its simple modules are written $S_{i}=k\left\{y^{i}\right\}$ for $0 \leq i \leq p-2$. (Here we use the notation that $R\{x, y, \ldots\}$ is the $R$-free module generated by $x, y, \ldots$.$) The summand L(1, i)$ is defined as $X\left(S\left(B, B, S_{i}\right)\right)=X_{S\left(B, B, S_{i}\right)}$ and $H^{*}(L(1, i)) \cong k[Y]\left\{y^{i}\right\}$ where $Y=y^{p-1}$. Hence we can decompose

$$
B\langle b\rangle \cong \vee_{i=0}^{p-2} L(1, i), \quad H^{*}(L(1, i)) \cong k[Y]\left\{y^{i}\right\} \quad \text { with } Y=y^{p-1} .
$$

The outer automorphism $\operatorname{Out}(A) \cong G L_{2}(k)$ and its simple modules are written as $S(A)^{i} \otimes \operatorname{det}^{j}$ for $0 \leq i \leq p-1,0 \leq j \leq p-2$. Here

$$
S^{i}(A)=H^{i}(A) \cong k\left\{y^{i}, y^{i-1} u, \ldots, u^{i}\right\}, \quad \operatorname{dim}\left(S(A)^{i}\right)=i+1
$$

and det is the determinate representation. Let us write $L(1, i)=$ $X_{S\left(P, \mathbb{Z} / p, S_{i}\right)}$, that is the image of the same named component by the 
split projections $A \rightarrow\left\langle a b^{\lambda}\right\rangle,\langle b\rangle 0 \leq \lambda \leq p-1$. Note that $\operatorname{Tr}_{A}^{P}(x)=0$ for all $x$. Hence we can show (e.g., Harris-Kuhn [Ha-Ku])

$$
B A \cong \vee_{i, q}(i+1) \tilde{X}_{i, q} \vee_{i \neq 0}(i+1) L(1, i)
$$

where $\tilde{X}_{i, q}=X_{S\left(A, A, S(A)^{i} \otimes \operatorname{det}^{q}\right)}$ for $0 \leq i \leq p-1,0 \leq q \leq p-2$, and $L(1, p-1)=L(1,0)$. However its decomposition of cohomology $H^{*}(A)$ is complicated.

For $j=(p-1)+i$ with $0 \leq i \leq p-2$, we consider

$$
\begin{gathered}
S(A)^{j}=k\left\{y^{j}, y^{j-1} u, \ldots, y^{p} u^{i-1}, T(A)^{i}, y^{i-1} u^{p}, \ldots, y u^{j-1}, u^{j}\right\} \\
\text { with } T(A)^{i}=k\left\{y^{p-1} u^{i}, y^{p-2} u^{i+1}, \ldots, y^{i} u^{p-1}\right\} .
\end{gathered}
$$

(Note $S(A)^{p-1}=T(A)^{0}$.) Let $d_{2}=y^{p} u-y u^{p} \in H^{*}(A)^{S L_{2}(k)}$ so that

$$
y^{j-1} u=y^{i-1} u^{p}, \ldots, y^{p} u^{i-1}=y u^{j-1} \bmod \left(d_{2}\right) .
$$

Then $S(A)^{j} \cong \tilde{S}(A)^{i} \oplus T(A)^{i} \bmod \left(d_{2}\right)$, where

$$
\tilde{S}(A)^{j}=k\left\{y^{j}\right\} \oplus k\left\{y^{j-1} u, \ldots, y^{p} u^{i-1}\right\} \oplus k\left\{u^{j}\right\} .
$$

We can see $\tilde{S}(A)^{j} \bmod \left(d_{2}\right)$ is a $G L_{2}(k)$-module, and hence $T(A)^{i} \cong$ $S(A)^{j} /\left(\tilde{S}(A)^{j},\left(d_{2}\right)\right)$ is also $G L_{2}(k)$-module (see [Hi-Ya1]). Moreover, we have

Lemma 4.1. (Lemma 4.3 in [Hi-Ya1]) There is an Out(E)-module isomorphism $T(A)^{i} \cong S(A)^{p-1-i} \otimes \operatorname{det}^{i}[2 i]$ where $[2 i]$ means the ascending degree $2 i$ operation.

Hereafter we use notation such that $A \ominus B=C$ means $A=B \oplus C$.

Theorem 4.2. We have an Out $(A)$-module decomposition

$$
H^{*}(A) \leftrightarrow k\left[d_{2}\right] \otimes\left(\left(k[\bar{C}] \otimes\left(\oplus_{i=0}^{p-2} S(A)^{i} \oplus T(A)^{i}\right)\right) \ominus k\{\bar{C}\}\right)
$$

where $\operatorname{Out}(A)$ acts trivially on $d_{2}$ and $\bar{C}$, and $|\bar{C}|=2(p-1)$.

Remark. The above theorem is proved in [Hi-Ya1], by using the map $q: E \rightarrow E /\langle c\rangle \rightarrow A$ where $E=p_{+}^{1+2}$ and $\langle c\rangle$ is its center (see $\S 6$ ). Then we can take

$$
g r H^{*}(A) \cong \operatorname{Im}\left(q^{*}\right) \oplus H^{*}(A)\left\{d_{2}\right\} \cong \operatorname{Im}\left(q^{*}\right) \otimes k\left[d_{2}\right] .
$$

The right hand side above is the module in the theorem. There is an element $C \in H^{2(p-1)}(E)$ such that $C \notin \operatorname{Im}\left(q^{*}\right)$ but $C x \in \operatorname{Im}\left(q^{*}\right)$ for $x \in H^{+}(A)$. We define $\bar{C} x=\left(q^{*}\right)^{-1}(C x)$. (Hence $\bar{C}$ itself does not exists in $g r H^{*}(A)$.) For example, $\bar{C} y=Y y, \bar{C} u=U u$ with $U=u^{p-1}$, and $\bar{C}^{2}=Y^{2}+U^{2}-Y U, \bar{C}^{3}=Y^{3}+U^{3}-Y^{2} U \bmod \left(d_{2}\right)$.

Let us write $\tilde{H}_{i, q} \cong(i+1) H^{*}\left(\tilde{Y}_{i, q}\right)$ is a summand of $H^{*}(A)$ which is the sum of all sub and quotient modules isomorphic to $S(A)^{i} \otimes \operatorname{det}^{q}$. Let us write $D_{2}=d_{2}^{p-1}$. Note that we use $k[\bar{C}] \ominus k\{\bar{C}\} \cong k\left[\bar{C}^{2}\right]\left\{1, \bar{C}^{3}\right\}$. 
Corollary 4.3. For $0 \leq q \leq p-2$, we have

$$
\tilde{H}_{i, q} \leftrightarrow\left\{\begin{array}{l}
k\left[\bar{C}^{2}, D_{2}\right]\left\{1, \bar{C}^{3}\right\} \quad \text { if } i=q=0, \\
k\left[\bar{C}^{2}, D_{2}\right]\left\{1, \bar{C}^{3}\right\}\left\{d_{2}^{q}\right\} \quad \text { if } i=0, q>0, \\
k\left[\bar{C}, D_{2}\right] \otimes\left(S(A)^{i} \otimes d_{2}^{q} \oplus T(A)^{p-1-i} \otimes d_{2}^{i+q}\right) \quad \text { otherwise. }
\end{array}\right.
$$

Since $H^{*}(L(1, i)) \subset H^{*}\left(\tilde{Y}_{i, 0}\right)$, (in fact $H^{*}(L(1, i)$ does not contain $\left.d_{2}^{q}\right)$, we have

$$
\tilde{Y}_{i, q} \cong\left\{\begin{array}{l}
\tilde{X}_{i, q} \quad \text { if } q \neq 0 \text { or }(i, q)=(0,0) \\
\tilde{X}_{i, 0} \vee L(1, i) \quad \text { if } q=0, i \neq 0 .
\end{array}\right.
$$

Let us write $\tilde{\mathbb{C}} \mathbb{B}=k\left[\bar{C}, D_{2}\right] \cong k\left[Y, D_{2}\right]$. Then we get

Theorem 4.4. ([Hi-Ya1]) We have $H^{*}\left(\tilde{X}_{0, q}\right) \cong \tilde{H}_{0, q}$. For $i \geq 1$, we have

$$
(i+1) H^{*}\left(\tilde{X}_{i, q}\right) \cong \begin{cases}\tilde{\mathbb{C}} \mathbb{B} \otimes\left(S(A)^{i}\left\{D_{2}\right\} \oplus T(A)^{p-1-i} \otimes d_{2}^{i}\right), & q=0 \\ \widetilde{\mathbb{C}} \mathbb{B} \otimes\left(S(A)^{i}\left\{d_{2}^{q}\right\} \oplus T(A)^{p-1-i} \otimes d_{2}^{i+q}\right) & q \neq 0 .\end{cases}
$$

In particular, the space $\tilde{X}_{p-1, q}$ (which is written as $L(2, q)$ e.g., in [Mi$\operatorname{Pr}]$ ),

$$
p H^{*}(L(2, q)) \cong\left\{\begin{array}{l}
\tilde{\mathbb{C}} \mathbb{B} \otimes\left(S(A)^{p-1}\left\{D_{2}\right\}\right), \quad q=0 \\
\tilde{\mathbb{C}} \mathbb{B} \otimes\left(S(A)^{p-1}\left\{d_{2}^{q}\right\}\right) \quad q \neq 0 .
\end{array}\right.
$$

Proof. We only need to prove the case $i \neq 0$ and $q=0$. First note

$$
(i+1) H^{*}\left(\tilde{X}_{i, 0}\right) \cong \tilde{H}_{i, q} \ominus(i+1) H^{*}(L(1,2) .
$$

Using Corollary 4.3, we can compute

$$
\begin{gathered}
(i+1) H^{*}\left(\tilde{X}_{i, 0}\right) \ominus\left(\tilde{\mathbb{C}} \mathbb{B} \otimes T(A)^{p-1-i} \otimes d_{2}^{i}\right) \\
\cong \tilde{\mathbb{C}} \mathbb{B} \otimes\left(S(A)^{i}\right) \ominus(i+1) k[Y]\left\{y^{i}\right\} \cong(\tilde{\mathbb{C}} \mathbb{B} \ominus k[Y]) \otimes S(A)^{i} .
\end{gathered}
$$

Here we identify $(i+1) k\left\{y^{i}\right\} \cong k\left\{y^{i}, y^{i-1} u, \ldots, u^{i}\right\} \cong S(A)^{i}$. The result follows from

$$
\tilde{\mathbb{C}} \mathbb{B} \ominus k[Y] \cong k\left[Y, D_{2}\right] \ominus k[Y] \cong k\left[Y, D_{2}\right]\left\{D_{2}\right\} \cong \tilde{\mathbb{C}} \mathbb{B}\left\{D_{2}\right\} .
$$

We recall the Dickson algebra $\mathbb{D} \mathbb{A}$, namely,

$$
\mathbb{D A}=k[y, u]^{G L_{2}(k)}=k\left[D_{1}, D_{2}\right]
$$

where $D_{1}=Y^{p}+V$ and $V=D_{2} / Y$ (see $\S 5,6$ below). Note that $\bar{C}^{p}=V+Y^{p}=D_{1} \bmod \left(d_{2}\right)$. Hence we can identify (as a free $\mathbb{D A}$ module)

$$
\tilde{\mathbb{C}} \mathbb{B}=\mathbb{D A}\left\{1, \bar{C}, \ldots, \bar{C}^{p-1}\right\} .
$$


Note $H^{*}\left(\tilde{X}_{0,0}\right) \not \mathbb{D} \mathbb{A}$. In fact, we have

\section{Lemma 4.5.}

$$
H^{*}\left(\tilde{X}_{0,0}\right) \cong k\left[\bar{C}^{2}, D_{2}\right]\left\{1, \bar{C}^{3}\right\} \cong \mathbb{D} \mathbb{A}\left\{1, D_{1} \bar{C}, \bar{C}^{2}, \ldots, \bar{C}^{p-1}\right\} .
$$

Proof. We see the last isomorphism

$$
\begin{aligned}
& \mathbb{C} \mathbb{B} \ominus k\{\bar{C}\} \otimes k\left[D_{2}\right] \cong \mathbb{D} \mathbb{A}\left\{1, \bar{C}, \ldots, \bar{C}^{p-1}\right\} \ominus k\{\bar{C}\} \otimes k\left[D_{2}\right] \\
& \cong \mathbb{D} \mathbb{A}\left\{1, \bar{C}^{2}, \ldots, C^{p-1}\right\} \oplus A \text { with } A=\mathbb{D} \mathbb{A}\{\bar{C}\} \ominus k\left[D_{2}\right]\{\bar{C}\} .
\end{aligned}
$$

Here $A \cong\left(\mathbb{D} \mathbb{A} \ominus k\left[D_{2}\right]\right)\{\bar{C}\} \cong \mathbb{D} \mathbb{A}\left\{D_{1} \bar{C}\right\}$. Thus we have the result.

Examples. For $p=3$, (see Corollary 5.2 in [Ya2]) we have

$$
\begin{gathered}
H^{*}\left(\tilde{X}_{0,0}\right) \cong \tilde{H}_{0,0} \cong H^{*}\left(\left(A: S D_{16}\right)\right) \cong H^{*}(A)^{S D_{16}} \\
\cong \mathbb{D} \mathbb{A}\left\{1, \tilde{C} D_{1}, \tilde{C}^{2}\right\} \cong \mathbb{D} \mathbb{A}\left\{1, \tilde{C}^{2}, \tilde{C}^{4}\right\}
\end{gathered}
$$

\section{METACYCLIC GROUPS FOR $p \geq 3$}

For $p \geq 5$, groups $P$ with $\operatorname{rank}_{p} P=2$ are classified by Blackburn (see Thomas [Th], Dietz-Priddy [Di-Pr] ). They are metacyclic groups, groups $C(r)$ and $G\left(r^{\prime}, e\right)$ (see sections 5,7 below for the definitions). In this section, we consider metacyclic $p$ groups $P$ for $p \geq 3$

$$
0 \rightarrow \mathbb{Z} / p^{m} \rightarrow P \rightarrow \mathbb{Z} / p^{n} \rightarrow 0 .
$$

These groups are represented as

$$
P=\left\langle a, b \mid a^{p^{m}}=1, a^{p^{m^{\prime}}}=b^{p^{n}},[a, b]=a^{r p^{\ell}}\right\rangle \quad r \neq 0 \bmod (p)
$$

It is known by Thomas [Th], Huebuschmann $[\mathrm{Hu}]$ that $H^{\text {even }}(P ; \mathbb{Z})$ is multiplicatively generated by Chern classes of complex representations. Let us write

$$
\left\{\begin{array}{l}
y=c_{1}(\rho), \quad \rho: P \rightarrow P /\langle a\rangle \rightarrow \mathbb{C}^{*} \\
v=c_{p^{m-\ell}}(\eta), \quad \eta=\operatorname{Ind}_{H}^{P}(\xi), \quad \xi: H=\left\langle a, b^{p^{m-\ell}}\right\rangle \rightarrow\langle a\rangle \rightarrow \mathbb{C}^{*}
\end{array}\right.
$$

where $\rho, \xi$ are nonzero linear representations. Then $H^{\text {even }}(P ; \mathbb{Z})$ is generated by

$$
y, c_{1}(\eta), c_{2}(\eta), \ldots, c_{p^{m-\ell}}(\eta)=v .
$$

(Lemma 3.5 and the explanation just before this lemma in [Ya1].) We can see (the last equation in the proof of Theorem 5.45 in [Ya1])

$$
c_{1}(\eta)=0, \ldots, c_{p^{m-\ell}-1}(\eta)=0 \quad \text { in } H^{*}(P)=H^{*}(P ; \mathbb{Z}) /(p, \sqrt{0}) .
$$

By using Quillen's theorem and the fact that $P$ has just one conjugacy class of maximal abelian $p$-subgroups, we can prove 
Theorem 5.1. (Theorem 5.45 in [Ya1]) For any metacyclic p-group $P$ in (5.1) with $p \geq 3$, we have a ring isomorphism

$$
H^{*}(P) \cong k[y, v], \quad|v|=2 p^{m-\ell}
$$

We now consider the stable splitting.

(I) Non split cases. For a non split metacyclic groups, it is proved that $B P$ itself is irreducible [Di].

(II) Split cases with $(\ell, m, n) \neq(1,2,1)$. We consider a split metacyclic group. it is written as

$$
P=M(\ell, m, n)=\left\langle a, b \mid a^{p^{m}}=b^{p^{n}}=1,[a, b]=a^{p^{\ell}}\right\rangle
$$

for $m>\ell \geq \max (m-n, 1)$.

The outer automorphism is the semidirect product

$$
\operatorname{Out}(P) \cong(p-\text { group }): \mathbb{Z} /(p-1) \text {. }
$$

The $p$-group acts trivially on $H^{*}(P)$, and $j \in \mathbb{Z} /(p-1)$ acts on $a \mapsto a^{j}$ and so acts on $H^{*}(P)$ as $j^{*}: v \mapsto j v$. There are $p-1$ simple $\mathbb{Z} /(p-1)$ modules $S_{i} \cong k\left\{v^{i}\right\}$. We consider the decomposition by idempotens for $\operatorname{Out}(P)$. Let us write $Y_{i}=e_{S_{i}} B P$ and

$$
H^{*}\left(Y\left(S_{i}\right)\right) \cong\left(\operatorname{dim}\left(S_{i}\right)\right) H^{*}\left(Y_{i}\right) \subset H^{*}(P)
$$

(in the notation $Y_{i}$ from Lemma 3.1). Hence we have the decomposition for $\operatorname{Out}(P)$-idempotents

$$
H^{*}\left(Y_{i}\right) \cong k[y, V]\left\{v^{i}\right\}, \quad V=v^{p-1} .
$$

We assume $P \neq M(1,2,1)$. By Dietz, we have splitting

$$
\text { (*) } \quad B P \cong \bigvee_{i=0}^{p=2} X_{i} \vee \bigvee_{i=0}^{p-2} \bar{L}(1, i)
$$

Here we write $X_{i}=e_{S\left(P, P, S_{i}\right)} B P$ identifying $S_{i}$ as the $A(P, P)$ simple module (but not the simple Out $(P)$-module).

The summand $\bar{L}(1, i)$ is defined as follows. (When $n=1, \bar{L}(1, i)=$ $L(1, i)$ defined in $\S 4$.) Recall that $H^{*}(\langle b\rangle) \cong k[y]$. The outer automorphism group is $\operatorname{Out}(\langle b\rangle) \cong\left(\mathbb{Z} / p^{n}\right)^{*}$ and its simple $k$ modules are $S_{i}^{\prime}=k\left\{y^{i}\right\}$ for $0 \leq i \leq p-2$. Hence we can decompose

$$
B\langle b\rangle \cong \vee_{i=0}^{p-2} \bar{L}(1, i), \quad H^{*}(\bar{L}(1, i)) \cong k[Y]\left\{y^{i}\right\} \quad \text { with } Y=y^{p-1}
$$

Next we consider $\bar{L}(1 . i)$ as a split summand in $B P$ as follows. (Consider the $A(P, P)$-simple module $S\left(P,\langle b\rangle, S_{i}^{\prime}\right)$.) Let $\Phi \in A(P, P)$ be the element defined by the map $\Phi: P \geq P \rightarrow\langle b\rangle \subset P$ which induced the isomorphism

$$
H^{*}(P) \Phi \cong H^{*}\left(\bigvee_{i=0}^{p-2} \bar{L}(1, i)\right) \cong k[y] \subset H^{*}\left(Y_{0}\right) .
$$


Thus we can show (since $k[y]$ is invariant under elements in $O u t(P)$ )

$$
(* *) \quad Y_{i} \cong\left\{\begin{array}{l}
X_{i} \quad i \neq 0 \\
X_{0} \vee \vee_{j=0}^{p-2} \bar{L}(1, j) \quad i=0 .
\end{array}\right.
$$

Remark. For groups $P, P^{\prime}$ with the same $m-\ell$, we have the isomorphism $H^{*}(P) \cong H^{*}\left(P^{\prime}\right)$ and the Burnside algebras act on the cohomology by the same way. For the splittings $X(P)$ and $X\left(P^{\prime}\right)$ (for $B P$ and $B P^{\prime}$ respectively), we have $H^{*}\left(X_{i}(P)\right) \cong H^{*}\left(X_{i}\left(P^{\prime}\right)\right)$. But when $P \neq P^{\prime}$, it is known from Nishida [Ni] that $X_{i}(P) \approx X_{i}\left(P^{\prime}\right)$, i.e. they are not stably homotopy equivalent. Similarly $\bar{L}(1, i)$ are different stable homotopy types when $n$ are different.

Theorem 5.2. Let $P$ be a split metacyclic group with $(\ell, m, n) \neq$ $(1,2,1)$. Then we have

$$
H^{*}\left(X_{i}\right) \cong \begin{cases}k[y, V]\left\{v^{i}\right\} & i \neq 0 \\ k[y, V]\{V\} & i=0 .\end{cases}
$$

Proof. For $i \neq 0$, we have $H^{*}\left(Y_{i}\right) \cong H^{*}\left(X_{i}\right)$. For $i=0$, we see

$$
\begin{gathered}
H^{*}\left(X_{0}\right) \cong H^{*}\left(Y_{0}\right) \ominus H^{*}\left(\vee_{j=0}^{p-2} L(1, j)\right) \\
\cong k[y, V] \ominus k[y] \cong k[y, V]\{V\} .
\end{gathered}
$$

(III) Split metacyclic group with $(\ell, m, n)=(1,2,1)$.

This case $P=p_{-}^{1+2}$ and its cohomology is the same as (II). But the splitting is given ([Di], $[\mathrm{Di}-\mathrm{Pr}])$

$$
B P \cong \bigvee_{i=0}^{p=2} X_{i} \vee \bigvee_{i=0}^{p-2} L(2, i) \vee \bigvee_{i=0}^{p-2} L(1, i) .
$$

Let $H=\left\langle b, a^{p}\right\rangle$ the maximal elementary abelian subgroup. The outer automorphism $O u t(H) \cong G L_{2}(k)$ and simple $G L_{2}(k)$-modules are written as $S(H)^{i} \otimes \operatorname{det}^{j}$. The summand $L(2, i)$ is defined as

$$
L(2, i)=X_{S\left(P, H, S(H)^{p-1} \otimes d e t^{i}\right)} .
$$

Here note $\left.v\right|_{H}=u^{p}-y^{p-1} u$ so that $\left.y v\right|_{H}=d_{2}$. This fact is proved by using the fact that $\left.v\right|_{H}$ invariant under the action $a^{*}: u \mapsto u+y, y \mapsto y$.

Of course there is no map $P \rightarrow H$. The space $L(2, i)$ is the transfer $(\operatorname{Tr}: B H \rightarrow B G)$ image of the same named summand of $B H$.

By using the double coset formula

$$
\left.\operatorname{Tr}_{H}^{P}\left(u^{p-1}\right)\right|_{H}=\sum_{i=0}^{p-1}(u+i y)^{p-1}=-y^{p-1}
$$


taking the generator $u$ in $H^{*}\left(\left\langle b, a^{p}\right\rangle\right) \cong k[y, u]$. The group $P$ has just one conjugacy class $H$ of the maximal abelian $p$-groups. Hence by Quillen's theorem, we have

$$
\operatorname{Tr}_{H}^{P}\left(\bar{C}^{i} d_{2}^{j} u^{p-1}\right)=-Y^{i}(y v)^{j} Y \quad \text { in } H^{*}(P)=H^{*}(P ; \mathbb{Z}) /(p, \sqrt{0}) .
$$

Next, we consider an element $\Phi \in A(P, P)$ defined by

$$
\Phi: P \geq H \stackrel{a^{p} \leftrightarrow b}{\cong} H \subset P .
$$

Then $\Phi\left(C^{i} d_{2}^{j}\right)=-C^{i} d_{2}^{j}$. (So $\left.\Phi^{2}\left(C^{i} d_{2}^{j}\right)=C^{i} d_{2}^{j}\right)$.) Here recall Theorem 4.4

$$
H^{*}(L(2, i)) \cong \begin{cases}\tilde{\mathbb{C}} \mathbb{B}\left\{\bar{C} d_{2}^{i}\right\} & i \neq 0 \\ \overline{\mathbb{C}} \mathbb{B}\left\{\bar{C} D_{2}\right\} & i=0 .\end{cases}
$$

Since $C^{i} d_{2}^{j}=C^{i} y^{j} v^{j} \in H^{*}\left(Y_{j}\right)$, we see $\Phi\left(H^{*}(L(2, j)) \subset H^{*}\left(Y_{j}\right)\right.$. Thus we have the isomorphism

$$
Y_{i} \cong\left\{\begin{array}{l}
X_{i} \vee L(2, i) \quad i \neq 0 \\
X_{0} \vee L(2,0) \vee \vee_{j=0}^{p-2} L(1, j) \quad i=0
\end{array}\right.
$$

To compute cohomology of irreducible components $X_{i}$ and $L(2, j)$, we recall the Dickson algebra

$$
\mathbb{D} \mathbb{A}=k[y, u]^{G L_{2}(\mathbb{Z} / p)} \cong k\left[D_{1}, D_{2}\right] \text { with } D_{1}=Y^{p}+V, D_{2}=Y V .
$$

We also write (see $\S 6$ bellow) the free $\mathbb{D} \mathbb{A}$-modules

$$
\begin{gathered}
\mathbb{C} \mathbb{A}=k[Y, V] \cong \mathbb{D} \mathbb{A}\left\{1, Y, \ldots, Y^{p}\right\}, \\
\mathbb{C} \mathbb{B}=k\left[Y, D_{2}\right] \cong \mathbb{D} \mathbb{A}\left\{1, Y, \ldots, Y^{p-1}\right\} .
\end{gathered}
$$

Hence $\mathbb{C} \mathbb{A} \cong \mathbb{D} \oplus \oplus \mathbb{C B}\{Y\}$.

Theorem 5.3. Let $P=M(1,2,1) \cong p_{-}^{1+2}$. Then we have

$$
H^{*}\left(X_{i}\right) \cong\left\{\begin{array}{l}
\mathbb{C} \mathbb{A}\left\{1, \ldots, \hat{y}^{i}, \ldots, y^{p-2}\right\}\left\{v^{i}\right\} \oplus \mathbb{D} \mathbb{A}\left\{d_{2}^{i}\right\} \quad i>0 \\
\mathbb{C} \mathbb{A}\left\{y, \ldots, y^{p-2}\right\}\{V\} \oplus \mathbb{D} \mathbb{A} \quad i=0 .
\end{array}\right.
$$

Proof. Let $i \neq 0$. We see

$$
H^{*}\left(Y_{i}\right) \cong k[y, V]\left\{v^{i}\right\} \cong \mathbb{C} \mathbb{A}\left\{1, y, \ldots, y^{p-2}\right\}\left\{v^{i}\right\} .
$$

The cohomology of the summand $X_{i}$ is

$$
\begin{gathered}
H^{*}\left(X_{i}\right) \cong H^{*}\left(Y_{i}\right) \ominus H^{*}(L(2, i)) \\
\cong \mathbb{C} \mathbb{A}\left\{v^{i}\right\}\left\{1, \ldots, y^{p-2}\right\} \ominus \mathbb{C B}\left\{Y d_{2}^{i}\right\} \\
\cong \mathbb{C} \mathbb{A}\left\{1, \ldots, \hat{y}^{i}, \ldots, y^{p-2}\right\}\left\{v^{i}\right\} \oplus\left(\mathbb{C} \mathbb{A}\left\{v^{i} y^{i}\right\} \ominus \mathbb{C} \mathbb{B}\left\{Y d_{2}^{i}\right\}\right) .
\end{gathered}
$$

Here $v^{i} y^{i}=d_{2}^{i}$ and $\mathbb{C} \mathbb{A} \cong \mathbb{D} \mathbb{A} \oplus \mathbb{C B}\{Y\}$, and we have the isomorphism in the theorem for $i \neq 0$. 
Next we consider in the case $i=0$. From Theorem 5.2, we see

$$
H^{*}\left(Y_{0}\right) \ominus H^{*}\left(\vee_{j} L(1, j) \cong k[y, v]\{V\} \cong \mathbb{C} \mathbb{A}\left\{1, \ldots, y^{p-2}\right\}\{V\} .\right.
$$

Hence we have

$$
\begin{gathered}
H^{*}\left(X_{0}\right) \cong H^{*}\left(Y_{0}\right) \ominus H^{*}\left(\vee_{j} L(1, j)\right) \ominus H^{*}(L(2,0)) \\
\cong \mathbb{C} \mathbb{A}\left\{1, y, \ldots, y^{p-2}\right\}\{V\} \ominus \mathbb{C B}\left\{Y D_{2}\right\} \cong \mathbb{C} \mathbb{A}\left\{y, \ldots, y^{p-2}\right\}\{V\} \oplus B
\end{gathered}
$$

where

$$
B=\mathbb{C} \mathbb{A}\{V\} \ominus \mathbb{C B}\left\{Y D_{2}\right\} \cong \mathbb{C} \mathbb{A} \ominus H^{*}(L(1,0)) \ominus H^{*}(L(2,0)) .
$$

We can see $B \cong \mathbb{D} \mathbb{A}$ by the following lemma.

Lemma 5.4. Let $M(2)=L(2,0) \vee L(1,0)$ (as the usual notation of the homotopy theory). Then we have

$$
H^{*}(M(2)) \cong \mathbb{C} \mathbb{B}\{Y\}, \quad \mathbb{C} \mathbb{A} \cong \mathbb{D} \mathbb{A} \oplus H^{*}(M(2)) .
$$

Proof. We can compute

$$
\begin{aligned}
& H^{*}(M(2)) \cong k[Y] \oplus \mathbb{C B}\left\{Y D_{2}\right\} \cong k[Y] \oplus k\left[Y, D_{2}\right]\left\{Y D_{2}\right\} \\
\cong & \left(k[Y] \oplus k\left[Y, D_{2}\right]\left\{D_{2}\right\}\right)\{Y\} \cong \mathbb{C B}\{Y\} \quad \text { (assumed *>0). }
\end{aligned}
$$

Since $\mathbb{C} \mathbb{A} \cong \mathbb{D} A \oplus \mathbb{C}\{Y\}$, we have the last isomorphism in this lemma.

\section{6. $C(r)$ GROUPS FOR $p \geq 3$}

The group $C(r), r \geq 3$ is the $p$-group of order $p^{r}$ such that

$$
C(r)=\left\langle a, b, a \mid a^{p}=b^{p}=c^{p^{r-2}}=1,[a, b]=c^{p^{r-3}}\right\rangle
$$

for $r \geq 3$ so that $C(3)=p_{+}^{1+2}$. Hence we have a central extension

$$
0 \rightarrow \mathbb{Z} / p^{r-2} \rightarrow C(r) \rightarrow \mathbb{Z} / p \times \mathbb{Z} / p \rightarrow 0 .
$$

For each $r \geq 3$, the cohomology $H^{*}(C(r))$ is isomorphic to $H^{*}(C(3))$. Denote $C(3)=p_{+}^{1+2}$ simply by $E$. The cohomology of $E$ is well known. In particular recall that $([\mathrm{Lw}],[\mathrm{Le} 1,2],[\mathrm{Te}-\mathrm{Ya}])$

$$
\text { (1) } H^{*}(E) \cong\left(k\left[y_{1}, y_{2}\right] /\left(y_{1}^{p} y_{2}-y_{1} y_{2}^{p}\right) \oplus k\{C\}\right) \otimes k[v] \text {. }
$$

Here $y_{1}$ (resp. $\left.y_{2}\right)$ is the first Chern class $c_{1}\left(e_{1}\right)$ (resp. $c_{1}\left(e_{2}\right)$ ) for the nonzero linear representation $e_{1}: E \rightarrow\langle a\rangle \rightarrow \mathbb{C}^{*}$ (resp. $e_{2}: E \rightarrow$ $\left.\langle b\rangle \rightarrow \mathbb{C}^{*}\right)$. The elements $C$ and $v$ are also represented by Chern classes

$$
c_{i}\left(\operatorname{Ind}_{A}^{E}(e)\right)= \begin{cases}v & \text { for } i=p \\ C & \text { for } i=p-1\end{cases}
$$

where $e: A \rightarrow\langle c\rangle \rightarrow \mathbb{C}^{*}$ is a non zero linear representation, for any maximal elementary abelian subgroup $A$. Hence $\left|y_{i}\right|=2,|C|=2(p-$ 
1), $|v|=2 p$. It is well known $C y_{i}=y_{i}^{p}, C^{2}=y_{1}^{2 p-2}+y_{2}^{2 p-2}-y_{1}^{p-1} y_{2}^{p-1}$. In this paper we write $y_{i}^{p-1}$ by $Y_{i}$, and $v^{p-1}$ by $V$.

From the Poincare series and formula (1), we get the another expression of $H^{*}(E)$ (Proposition 9 in [Gr-Le], or [Ya2])

$$
H^{*}(E) \cong k[C, v]\left\{y_{1}^{i} y_{2}^{j} \mid 0 \leq i, j \leq p-1,(i, j) \neq(p-1, p-1)\right\} .
$$

Let us write $(\mathbb{Z} / p)^{2}$ by $A$ simply. The $E$ conjugacy classes of $A$ subgroups are written by

$$
A_{i}=\left\langle c, a b^{i}\right\rangle \text { for } 0 \leq i \leq p-1, \quad A_{\infty}=\langle c, b\rangle .
$$

For $A=A_{i}$ some $i$, if we take $y, u$ as $H^{*}(A) \cong k[y, u]$, then $\left.C\right|_{A}=Y$ and $\left.v\right|_{A}=u^{p}-y^{p-1} u$. The transfer map is given by $\operatorname{Tr}_{A_{0}}^{E}(y)=0$ and

$$
\text { (3) } \operatorname{Tr}_{A_{j}}^{E}\left(u^{i}\right)=\left\{\begin{array}{l}
\left(j y_{1}+y_{2}\right)^{p-1}-C \quad \text { if } i=p-1 \\
0 \quad \text { for } i<p-1
\end{array}\right.
$$

(for $j=\infty$, we have $\operatorname{tr}_{A_{\infty}}^{E}\left(u^{p-1}\right)=y_{1}^{p-1}-C$ ).

We first consider the Out $(E)$-module decomposition of $H^{*}(E)$. Recall that $\operatorname{Out}(E) \cong \operatorname{Out}(A) \cong G L_{2}\left(\mathbb{F}_{p}\right)$. The simple modules of $G=G L_{2}\left(\mathbb{F}_{p}\right)$ are well known. Let us think of $A$ as the natural twodimensional representation, and det the determinant representation of $G$. Then there are $p(p-1)$ simple $k[G]$-modules given by $S(A)^{i} \otimes(\text { det })^{q}$ for $0 \leq i \leq p-1,0 \leq q \leq p-2$ where

$$
S(A)^{i}=k\left\{y_{1}^{i}, y_{1}^{i-1} y_{2},, \ldots, y_{2}^{i}\right\} \cong H^{2 i}(E) .
$$

(This $S(A)^{i}$ is isomorphic to $S(A)^{i}$ in $\S 4$, but take generators $y_{1}$ (resp. $\left.y_{2}\right)$ for $y$ (resp. $u$ ).

Recall that $k\{v\} \cong \operatorname{det}$ as $O u t(E)$-modules. Note that

$$
\mathbb{C} \mathbb{A}=k[C, V] \cong H^{*}(E)^{\text {Out }(E)} .
$$

For $j=(p-1)+i$ with $0 \leq i \leq p-2$. Write it

$$
H^{i}(E) \supset T(A)^{i}, \quad T(A)^{i}=k\left\{y_{1}^{p-1} y_{2}^{i}, y_{1}^{p-2} y_{2}^{i+1}, \ldots, y_{1}^{i} y_{2}^{p-1}\right\} .
$$

(Note $S(A)^{p-1}=T(A)^{0}$.) Using the the relation $d_{2}=y_{1}^{p} y_{2}-y_{1} y_{2}^{p}=$ 0 in $H^{*}(E)$, we can consider $T(A)^{i}$ is an $O u t(E)$-module such that $T(A)^{i} \cong S(A)^{p-1-i} \otimes \operatorname{det}^{i}[2 i]$ from Lemma 4.1. In fact, from (2), we also have

$$
H^{*}(E) \cong k[C, v] \otimes\left(\oplus_{i=0}^{p-2}\left(S(A)^{i} \oplus T(A)^{i}\right)\right)
$$

Hence we have

Theorem 6.1. (Theorem 4.4 in [Hi-Ya1]) There is a decomposition of Out(E)-module such that

$$
H^{*}(E) \leftrightarrow \mathbb{C} \mathbb{A} \otimes\left(\oplus_{q=0}^{p-2} \oplus_{i=0}^{p-2}\left(S(A)^{i} \otimes v^{q} \oplus T(A)^{i} \otimes v^{q}\right)\right)
$$


where $S(A)^{i} \otimes v^{q} \cong S(A)^{i} \otimes \operatorname{det}^{q}, \quad T(A)^{i} \otimes v^{q} \cong S(A)^{p-1-i} \otimes \operatorname{det}^{i+q}[2 i]$.

Let us write by $H_{i, q}$ the summand of $H^{*}(E)$ which is a sum of the all (sub and quotient) modules isomorphic to $S(A)^{i} \otimes \operatorname{det}^{q}$. (In the notation in $\S 3, H_{i, j}^{*} \cong(i+1) H^{*}\left(Y_{i, j}\right)$.)

Corollary 6.2. We have the Out $(E)$-module decomposition

$$
H_{i, q} \leftrightarrow \begin{cases}\mathbb{C} \mathbb{A} \otimes v^{q} \quad \text { for } i=0, \\ \mathbb{C} \mathbb{A} \otimes T(A)^{0} \otimes v^{q} \quad\left(T(A)^{0}=S(A)^{p-1}\right) & \text { for } i=p-1, \\ \mathbb{C} \mathbb{A} \otimes\left(S(A)^{i} \otimes v^{q} \oplus T(A)^{p-1-i} \otimes v^{i+q}\right) & \text { otherwise. }\end{cases}
$$

(I) $P=C(r), r>3$.

By Dietz and Priddy, the stable splitting is known. The splitting is given as

$$
B P \cong \vee(i+1) X_{i, q} \vee(q+1) L(1, q) \vee p L(1, p-1)
$$

where $0 \leq i \leq p-1,0 \leq q \leq p-2$ and $L(1, p-1)=L(1,0)$. Transfers from proper subgroups are always zero when $r>3$. We have

$$
Y_{i, q}=\left\{\begin{array}{l}
X_{i, q} \quad q \neq 0 \\
X_{i, 0} \vee L(1, i) \quad q=0 .
\end{array}\right.
$$

Theorem 6.3. Let $P=C(r)$ and $r \geq 4$. Then

$$
(i+1) H^{*}\left(X_{i, q}\right) \cong\left\{\begin{array}{l}
H_{i, q} \quad \text { if } q \neq 0 \\
\left.\mathbb{C} \mathbb{A} \otimes\left(S^{i}(A)\right\}\{V\} \oplus T^{p-1-i}(A) v^{i}\right) \quad q=0, i \neq p-1 \\
\mathbb{C} \mathbb{A} \otimes S^{p-1}(A)\{V\} \quad q=0, i=p-1 .
\end{array}\right.
$$

Proof. We only need to prove $q=0$. Note that $\mathbb{C} \mathbb{A} \cong k[C, V] \cong$ $k[C] \oplus \mathbb{C} \mathbb{A}\{V\}$. So we have $\mathbb{C} \mathbb{A} \ominus k[C] \cong \mathbb{C} \mathbb{A}\{V\}$. Then we can compute as

$$
\begin{gathered}
\mathbb{C} \mathbb{A} \otimes S^{i}(A) \ominus(i+1) k[C]\left\{y^{i}\right\} \\
\cong \mathbb{C} \mathbb{A}\{V\}\left\{y_{1}^{i}, y_{1}^{i-1} y_{2}, \ldots, y_{2}^{i}\right\} \cong \mathbb{C} \mathbb{A} \otimes S(A)^{i}\{V\} .
\end{gathered}
$$

Using this and

$$
(i+1) H^{*}\left(X_{i, 0}\right) \cong(i+1) H^{*}\left(Y_{i, 0}\right) \ominus(i+1) H^{*}(L(1, i))
$$

we can get the theorem (for $q=0$ ).

(II) $C(3)=p_{+}^{1+2}$.

In this case, the decomposition of cohomology is given in [Hi-Ya1] but it is quite complicated. By Dietz-Priddy, the splitting is given as $B P \cong \vee(i+1)_{i, q} X_{i, q} \vee \vee_{k}(p+1) L(2, q) \vee_{q}(q+1) L(1, q) \vee p L(1, p-1)$ 
where $0 \leq i \leq p-1$ and $0 \leq q \leq p-2$. The different places from $r \geq 4$ are the existence of $L(2, q)$ which are induced from the transfer (see $\S 9$ in [Hi-Ya1] for details).

Lemma 6.4. We have the isomorphisms

$$
\begin{gathered}
(p+1) \oplus_{q=1}^{p-1} H^{*}(L(2, q)) \cong \mathbb{C B} \otimes\left(\oplus_{q=1}^{p-1} \oplus_{j \in \mathbb{F}_{p} \cup \infty}(1)_{q, j}\right) \\
\cong \mathbb{C B} \otimes\left(\oplus_{q=1}^{p-1}(2)_{q}\right) \text { where } \\
(1)_{q, j}=k\left\{\operatorname{Tr}_{A_{j}}^{E}\left(u^{p-1}\right) d_{2}\left(A_{j}\right)^{q}\right\} \cong k\left\{\left(\left(j y_{1}+y_{2}\right)^{p-1}-C\right) d_{2}\left(A_{j}\right)^{q}\right\}, \\
(2)_{q}=\left\{\begin{array}{l}
\left(S(A)^{q}\left\{C \otimes v^{q}\right\} \oplus T(A)^{q} \otimes v^{q}\right) \quad 1 \leq q \leq p-2 \\
\left(S(A)^{p-1} \oplus k\{C\}\right)\left\{D_{2}\right\} \quad q=p-1
\end{array}\right.
\end{gathered}
$$

where $d_{2}\left(A_{j}\right)=v\left(y_{1}+j y_{2}\right)$.

Outline of Proof. (See $\S 9$ in [Hi-Ya1] for details.) Let $x \in(1)_{q, j} \subset$ $\left(k\{C\} \oplus S(A)^{p-1}\right) \otimes d_{2}^{q}$. Then for $\mu, \lambda_{i} \in \mathbb{Z} / p$, the element $x$ is written as

$$
\begin{gathered}
\mu C \otimes d_{2}^{q}+\sum_{i} \lambda_{i} y_{1}^{i} y_{2}^{p-1-i} \otimes d_{2}^{q} \\
=\mu C y_{1}^{q} \otimes v^{q}+\sum_{i} \lambda_{i} y_{1}^{i+q} y_{2}^{p-1-i} \otimes v^{q} \\
\in\left(S(A)^{q}\left\{C \otimes v^{q}\right\} \oplus T(A)^{q} \otimes v^{q}\right) \subset H_{q, q} \oplus H_{p-1-q, 2 q} .
\end{gathered}
$$

The last inclusion follows from $T^{q} \otimes v^{q} \cong S(A)^{p-1-q} \otimes d e t^{q+q}$ as $O u t(P)$ modules. Hence we see $\bigoplus_{j \in \mathbb{F}_{p} \cup \infty}(1)_{q, j} \subset(2)_{q}$. Since

$$
(1)_{q, j} \mid A_{k}=\left\{\begin{array}{l}
\left(y_{1}+j y_{2}\right)^{p-1} d_{2}\left(A_{j}\right)^{q} \quad j=k \\
0 \quad \text { otherwise, }
\end{array}\right.
$$

we have $\operatorname{dim}\left(\oplus_{j \in \mathbb{F}_{p} \cup \infty}(1)_{q}\right) \geq p+1$. Of course (from Theorem 6.1), we have $\operatorname{dim}(2)_{q}=p+1$. Therefore we show that $\bigoplus_{j \in \mathbb{F}_{p} \cup \infty}(1)_{q}=(2)_{q}$.

Note that $(p+1) H^{*}(L(2, q)) \subset H_{q, q} \oplus H_{p-1-q, i+q}$, however each $H^{*}(L(2, q))$ is contained in either $H^{*}\left(Y_{q, q}\right)$ or $H^{*}\left(Y_{p-1-i, i+q}\right)$.

Theorem 6.5. The Out $E)$-module decomposition

$$
H^{*}(E) \leftrightarrow \oplus_{i, q} H_{i, q} \cong \oplus_{i, q} \mathbb{C} \mathbb{A} \otimes\left(S(A)^{i} \otimes v^{q} \oplus T(A)^{p-1-i} \otimes v^{2 q}\right)
$$

gives simple $A(E, E)$-modules decomposition by each of the following sums of Out $(E)$-simple modules

$$
\text { (1) } S(A)^{p-1} \oplus k\{C\}
$$

$$
\text { (2) } S(A)^{q}\{C\} \otimes v^{q} \oplus T(A)^{q} \otimes v^{q}
$$

as one $A(E, E)$-simple module for $1 \leq q \leq p-2$. That is
$(1) \cong S\left(E, \mathbb{Z} / p, \operatorname{det}^{0}\right)[2 p-2]$
$(2) \cong S\left(E, A, S(A)^{p-1} \otimes \operatorname{det}^{q}\right)[2(p-1) q]$. 
Outline of Proof. We prove that (1) is a simple $A(E, E)$-module. We consider $\Phi_{1}, \Phi_{2} \in A(E, E)$

$$
\begin{gathered}
\Phi_{1}: P>A_{0} \rightarrow\langle c\rangle \stackrel{w}{\cong}\langle a\rangle \subset A_{0} \subset P, \\
\Phi_{2}: P>A_{\infty} \rightarrow\langle c\rangle \stackrel{w}{\cong}\langle b\rangle \subset A_{\infty} \subset P .
\end{gathered}
$$

Then we see $\left(\Phi_{1}-\Phi_{2}\right)(C)=y_{1}^{p-1}-y_{2}^{p-1} \in S^{p-1}(A)$, and

$$
\Phi_{1}\left(y_{1}^{p-1}\right)=y_{2}^{p-1}-C \in S^{p-1}(A) \oplus k\{C\} .
$$

Let $B=\left(S^{p-1}(A) \oplus k\{C\}\right)$. Then from the first equation, $B / k\{C\}$ is not an $A(P, P)$-module. From the second one, we see that $B / S^{p-1}(A)$ is also not an $A(P, P)$-module. Hence $B$ is a simple $A(P, P)$-module.

The fact that $(2)$ is isomorphic to a $A(P, P)$ simple module is proved similarly using $\Phi_{1}^{\prime}$ and $\Phi_{2}^{\prime}$ defined by

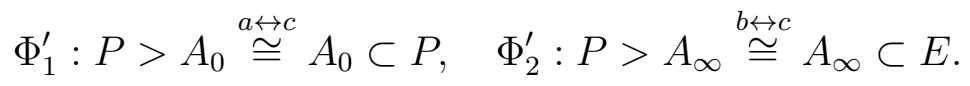

Note that $\operatorname{dim}(2)=(q+1)+(p-1-q+1)=p+1$. In fact, this is the number of $L(2, q)$ in $B E$.

Let $i=0$ or $p-1$. Then we see

$$
Y_{i, q} \cong\left\{\begin{array}{l}
X_{i, q} \quad q \neq 0 \\
X_{i, 0} \vee L(2,0) \vee L(1,0) \quad q=0 .
\end{array}\right.
$$

Using this we can prove

Theorem 6.6. (Corollary 10.7 in [Hi-Ya1]) We have for $1 \leq q \leq p-2$,

$$
\begin{gathered}
H^{*}\left(X_{0,0}\right) \cong \mathbb{D} \mathbb{A}, \quad p H^{*}\left(X_{p-1,0}\right) \cong \mathbb{D} \mathbb{A} \otimes S(A)^{p-1}\{V\}, \\
H^{*}\left(X_{0, q}\right) \cong H_{0, q} \cong \mathbb{C} \mathbb{A}\left\{v^{q}\right\}, \quad p H^{*}\left(X_{p-1, q}\right) \cong H_{p-1, q} \cong \mathbb{C} \mathbb{A}\left\{S(A)^{p-1} \otimes v^{q}\right\} .
\end{gathered}
$$

Proof of the first isomorphism. Recall $H^{*}(L(2,0) \vee L(1,0)) \cong \mathbb{C B}\{C\}$ from Lemma 5.4. Hence we see

$$
H^{*}\left(X_{0,0}\right) \cong \mathbb{C} \mathbb{A} \ominus \mathbb{C} \mathbb{B}\{C\} \cong \mathbb{D} \mathbb{A} .
$$

The other cases are proved similarly.

Examples. See $\S 6$ in [Ya2] for $p=3$ case. For the sporadic simple group $J_{4}$ and the twisted Chevalley group ${ }^{2} F_{4}^{\prime}$, we have the isomorphisms

$$
\begin{gathered}
H^{*}\left(J_{4}\right) \cong H^{*}\left(X_{0,0}\right) \cong \mathbb{D} \mathbb{A}, \quad(\text { Green }[G r]) \\
H^{*}\left({ }^{2} F_{4}^{\prime}\right) \cong H^{*}\left(X_{0,0} \vee X_{2,0}\right) \cong \mathbb{D} \mathbb{A}\{1, Y V\}, \\
H^{*}(E)^{S D_{16}} \cong H^{*}\left(X_{0,0} \vee L(2,0) \vee L(1,0)\right) \cong \mathbb{C} \mathbb{A},
\end{gathered}
$$


Theorem 6.7. (Corollary 10.8 in [Hi-Ya1]) Let $1 \leq i \leq p-2$. Let us write simply

$$
S=S(A)^{i} \otimes v^{q}, \quad T=T(A)^{p-1-i} \otimes v^{i+q} .
$$

Then we have the isomorphism

$$
(1+i) H^{*}\left(X_{i, q}\right) \cong\left\{\begin{array}{l}
\mathbb{D} \mathbb{A} \otimes(S \oplus T\{V\}) \text { if } i=q \neq 0,3 q \equiv 0(\bmod (p-1)) \\
\mathbb{D} \mathbb{A} \otimes S \oplus \mathbb{C} \mathbb{A} \otimes T \text { if } i=q, 3 q \neq \equiv \\
\mathbb{C} \mathbb{A} \otimes S \oplus \mathbb{D} \mathbb{A} \otimes T\{V\} \text { if } q \equiv-2 i \neq 0,3 i \neq 0 \\
\mathbb{C} \mathbb{A} \otimes S\{V\} \oplus \mathbb{D} \mathbb{A} \otimes T\{V\} \text { if } q \equiv 0,2 i \equiv 0 \\
\mathbb{C} \mathbb{A} \otimes S\{V\} \oplus \mathbb{C} \mathbb{A} \otimes T \text { if } q \equiv 0,2 i \neq 0 \\
H_{i, q} \cong \mathbb{C} \mathbb{A} \otimes(S \oplus T) \text { otherwise. }
\end{array}\right.
$$

Outline of Proof. From the proof of Lemma 5.5, we see

$$
\oplus_{j} \operatorname{Tr}_{A_{j}}^{E}\left(H^{*}(L(2, q)) \subset H_{q, q} \oplus H_{p-1-q, 2 q} .\right.
$$

We prove the first isomorphism. Suppose $3 q \equiv 0, q \not \equiv 0 \bmod (p-1)$. Then ( see the proof of Lemma 6.4)

$$
\oplus_{j} \operatorname{Tr}_{A_{j}}^{E}\left(H^{*}(L(2,2 q)) \subset H_{2 q, 2 q} \oplus H_{q, q}\right.
$$

since $H_{p-1-2 q, 4 q}=H_{q, q}$. Using this we can prove

$$
Y_{q, q} \cong X_{q, q} \vee L(2, q) \vee L(2,2 q)
$$

Hence we can compute

$$
\begin{gathered}
(q+1) H^{*}\left(X_{q, q}\right) \cong\left(\mathbb{C} \mathbb{A} \otimes S \ominus(q+1) \mathbb{C B}\left\{C d^{q}\right\}\right) \\
\oplus\left(\mathbb{C} \mathbb{A} \otimes T \ominus(q+1) \mathbb{C B}\left\{C d^{2 q}\right\}\right) .
\end{gathered}
$$

Here $d^{q}=y^{q} v^{q} \in S=S^{q}(A)\left\{v^{q}\right\}$ and $C d_{2}^{2 q}=\left(C y^{2 q}\right) v^{2 q} \in T=$ $T(A)^{2 q}\left\{v^{2 q}\right\}$. Hence

$$
\mathbb{C} \mathbb{A} \otimes S \ominus(q+1) \mathbb{C B}\left\{C d^{q}\right\} \cong(\mathbb{C} \mathbb{A} \ominus \mathbb{C B}\{C\})\left\{S^{q}(A) v^{q}\right\} \cong \mathbb{D} \mathbb{A} \otimes S .
$$

$$
\mathbb{C} \mathbb{A} \otimes T \ominus(q+1) \mathbb{C B}\left\{C d^{2 q}\right\} \cong(\mathbb{C} \mathbb{A} \ominus \mathbb{C B})\left\{T^{2 q} v^{2 q}\right\} \cong \mathbb{D} \mathbb{A}\left\{C^{p}\right\} \otimes T .
$$

Note $D_{1}=C^{p}+V$. Thus we can prove the first isomorphism. The other isomorphisms are proved similarly.

We write down here the splitting in the all cases. 
Corollary 6.8. For $1 \leq i \leq p-2$, there are stable homotopy equivalences

$$
Y_{i, q} \cong\left\{\begin{aligned}
& X_{q, q} \vee L(2, q) \vee L(2,2 q) \\
& \text { if } i=q \neq 0,3 q \equiv 0(\bmod (p-1)) \\
& X_{q, q} \vee L(2, q) \quad \text { if } i=q, 3 q \not \equiv 0 \\
& X_{i,-2 i} \vee L(2,-i) \quad \text { if } q \equiv-2 i \neq 0,3 i \neq 00 \\
& X_{i, 0} \vee L(1, i) \vee L(2,-i) \text { if } q \equiv 0,2 i \equiv 0 \\
& X_{i, 0} \vee L(1, i) \text { if } q \equiv 0,2 i \neq \equiv 0 \\
& X_{i, q} \text { otherwise. }
\end{aligned}\right.
$$

Example. When $p=7$ (see $\S 9$ in [Ya2]), we see the cohomology

$$
\begin{gathered}
H^{*}\left(X_{0,0}\right) \cong \mathbb{D} \mathbb{A}, H^{*}\left(X_{6,0}\right) \cong \mathbb{D} \mathbb{A}\left\{a^{3}\right\}, H^{*}\left(X_{4,4}\right) \cong \mathbb{D} \mathbb{A}\left\{a^{2}, a^{4}\right\}, \\
H^{*}\left(X_{2,2}\right) \cong \mathbb{D} \mathbb{A}\left\{a, a^{5}\right\} .
\end{gathered}
$$

where $a=s^{2} \otimes v^{2} \in S(2,2), a^{5}=s^{10} \otimes v^{10}=s^{10} \otimes v^{4} V \in T(2,2), \ldots$ and where $S(i, q)=S, T(i, q)=T$ in the preceding theorem. Here $a^{6}=D_{2}^{2}$ (page 416 in [Ya2]). Thus we see the cohomology of the exotic finite 7-groups (see $\S 9$ in [Ya2]) found by Ruiz and Viruel [Ru-Vi]

$$
\begin{gathered}
H^{*}\left(R V_{3}\right) \cong H^{*}\left(X_{0,0} \vee X_{4,4}\right) \cong \mathbb{D} \mathbb{A}\left\{1, a^{2}, a^{4}\right\}, \\
H^{*}\left(R V_{2}\right) \cong H^{*}\left(X_{0,0} \vee X_{4,4} \vee X_{6,0} \vee X_{2,2}\right) \cong \mathbb{D} \mathbb{A}\left\{1, a, a^{2}, a^{3}, a^{4}, a^{5}\right\} \\
H^{*}\left(R V_{1}\right) \cong H^{*}\left(X_{0,0} \vee X_{6,0} \vee X_{4,4}\right) \cong \mathbb{D} \mathbb{A}\left\{1, a^{2}, a^{3}, a^{4}\right\} .
\end{gathered}
$$

Therefore there does not exist even a 7-local finite group $G$ such that $H^{*}(G) \cong \mathbb{D} \mathbb{A}$.

$$
\text { 7. } G(r, e) \text { FOR } p \geq 5
$$

For $p \geq 5$, groups $P$ with $\operatorname{rank}_{p} P=2$ are classified by Blackburn (see Thomas [Th], Dietz-Priddy [Di-Pr], [Ya1] ). They are metacyclic groups, groups $C(r)$ and $G\left(r^{\prime}, e\right)$. Throughout this section, we assume $p \geq 5$.

The group $G=G(r, e), r \geq 4$ (and $e$ is 1 or a quadratic non residue modulo $p$ ) is defined as

$$
\left\langle a, b, c \mid a^{p}=b^{p}=c^{p^{r-2}}=[b, c]=1,\left[a, b^{-1}\right]=c^{e p^{r-3}},[a, c]=b\right\rangle .
$$

The subgroup $\left\langle a, b, c^{p}\right\rangle$ is isomorphic to $C(r-1)$. Hence we have the extension

$$
1 \rightarrow C(r-1) \rightarrow G(r, e) \rightarrow \mathbb{Z} / p \rightarrow 0 .
$$

Of course $E=C(3) \subset C(r-1) \subset G(r, e)$. By [Ya1], we have an isomorphism

$$
H^{*}(G(r, e)) \cong H^{*}(E)^{\langle c\rangle} .
$$


Indeed, in Theorem 5.29 in [Ya1], we see $H^{\text {even }}(G ; \mathbb{Z}) \cong\left(Y_{1} \oplus Y_{w} \oplus\right.$ $\left.C^{\prime}\right) \otimes C_{p}^{\prime}$ and in $(5.5)$, we see $H^{\text {even }}(E ; \mathbb{Z})^{\langle c\rangle} \cong\left(Y_{1} \oplus Y_{w} \oplus C\right) \otimes C_{p}$. Here we can show

$$
C_{p} / p \cong C_{p}^{\prime} / p, \quad C / p \cong\left\{\begin{array}{l}
C^{\prime} / p \quad \text { for } r=4 \\
C^{\prime} /\left(p, c_{1}\right), \quad\left(c_{1}: \text { nilpotent }\right) \text { for } r \geq 5 .
\end{array}\right.
$$

The invariant ring $H^{*}(C(3))^{\langle c\rangle}$ is multiplicatively generated by

$$
y_{1}, C, v, y_{2}^{i} w \quad \text { where } w=y_{2}^{p}-y_{1}^{p-1} y_{2}, \quad 0 \leq i \leq p-3
$$

since $c^{*}: y_{2} \mapsto y_{2}+y_{1}$ and $C^{2}=Y_{1}^{2}+y_{2}^{p-2} w$. Hence we have

Lemma 7.1. We have an isomorphism

$$
\text { (1) } H^{*}(G(r, e)) \cong\left(k\left[y_{1}\right] \oplus k\left[y_{2}\right]\{w\} \oplus k\{C\}\right) \otimes k[v]
$$

where the multiplications are given by $y_{1} w=0, C y_{1}=y_{1}^{p}, w^{2}=y_{2}^{p} w$

and $C w=y_{2}^{p-1} w$, Thus we also have the isomorphism

(2) $H^{*}(G(r, e)) \cong \mathbb{C} \mathbb{A}\left(\oplus_{q=0}^{p-2}\left(k\left\{1, y_{1}, \ldots, y_{1}^{p-1}\right\}\left\{v^{q}\right\} \oplus k\left\{1, y_{2}, \ldots, y_{2}^{p-3}\right\}\left\{w v^{q}\right\}\right)\right.$.

Here we note that

$$
\begin{gathered}
H^{*}(E)^{\langle c\rangle} \cap \oplus_{i=0}^{p-2} S(A)^{i} \cong k\left\{1, y_{1}, \ldots, y_{1}^{p-2}\right\}, \\
H^{*}(E)^{\langle c\rangle} \cap \oplus_{i=0}^{p-2} T(A)^{i} \cong k\left\{y_{1}^{p-1}\right\} \oplus k\left\{1, y_{2}, \ldots, y_{2}^{p-3}\right\}\{w\} .
\end{gathered}
$$

Let us write $w_{i+1}=y_{2}^{i} w\left(\right.$ so $\left.w_{1}=w\right)$ and

$$
S(G)=k\left\{1, y_{1}, \ldots, y_{1}^{p-2}\right\}, \quad T(G)=k\left\{y_{1}^{p-1}, w_{1}, \ldots, w_{p-2}\right\}
$$

so that $H^{*}(G(r, e)) \cong \mathbb{C} \mathbb{A} \otimes\left(\oplus_{i}(S(G) \oplus T(G))\left\{v^{i}\right\}\right)$.

For groups $G^{\prime}=G(r, e), E, \ldots$, let us write by $Y_{i, j}\left(G^{\prime}\right)\left(\right.$ and $\left.X_{i, j}\left(G^{\prime}\right)\right)$ the decomposition component for $B G^{\prime}$. Then from Corollary 6.2, we have

Lemma 7.2. We have additively

$$
\begin{aligned}
H^{*}(G(r, e)) \cong \oplus_{i, q} H^{*}\left(Y_{i, q}(E)\right) \quad \text { with } \\
H^{*}\left(Y_{i, q}(E)\right) \cong\left\{\begin{array}{l}
\mathbb{C} \mathbb{A} \otimes v^{q} \quad \text { for } i=0, \\
\mathbb{C} \mathbb{A} \otimes k\left\{y_{1}^{p-1} \otimes v^{q}\right\} \quad \text { for } i=p-1, \\
\mathbb{C} \mathbb{A} \otimes\left(k\left\{y_{1}^{i} \otimes v^{q}, w_{p-1-i} \otimes v^{i+q}\right\}\right) \text { otherwise }
\end{array}\right.
\end{aligned}
$$

where $0 \leq i \leq p-1$ and $0 \leq q \leq p-2$.

The outer automorphism is $\operatorname{Out}(P) \cong(p-$ group $):(\mathbb{Z} / 2 \times \mathbb{Z} /(p-1))$ (see $[\mathrm{Di}-\mathrm{Pr}]$ for details). Here the action $i \in \mathbb{Z} / 2$ induces $i: a \mapsto a^{-1}$ and $k \in \mathbb{Z} /(p-1)$ induces $k: c \mapsto c^{k}$. Hence

$$
i^{*}:\left\{\begin{array}{l}
y_{1} \mapsto-y_{1} \\
y_{2} \mapsto-y_{2},
\end{array} \quad k^{*}:\left\{\begin{array}{l}
v \mapsto k v \\
y_{2} \mapsto k y_{2}
\end{array}\right.\right.
$$


All simple $\mathbb{Z} / 2 \times \mathbb{Z} /(p-1)$-modules are represented as

$$
k\left\{v^{i}\right\}, \quad k\left\{y_{1} v^{i}\right\} \quad 0 \leq i \leq p-2 .
$$

Using this and Lemma 7.2 (2), we get

Lemma 7.3. Let $P=G(r, e)$ with $r \geq 4$. Then we have Out $(P)$ module decomposition

$$
H_{i, q} \leftrightarrow H^{*}\left(Y_{i, q}(P)\right) \cong\left\{\begin{array}{l}
\oplus_{j=\text { even }} H^{*}\left(Y_{j, q}(E)\right) \text { if } i=0 \\
\bigoplus_{j=\text { odd }} H^{*}\left(Y_{j, q}(E)\right) \text { if } i=1
\end{array}\right.
$$

where $0 \leq i \leq 1,0 \leq j \leq p-1$ and $0 \leq q \leq p-2$.

(I) The case $P=G(r, e)$ and $r>4$.

The stable splitting is given by Dietz-Priddy [Di-Pr]

$$
B G(r, e) \cong \vee_{i, q} X_{i, q}(G(r, e)) \vee \vee_{q} X_{p-1, q}(C(r-1)) \vee \vee_{q} L(1, q)
$$

where $i \in \mathbb{Z} / 2$ and $0 \leq q \leq p-2$.

From Theorem 6.3, (for $r-1 \geq 4$ ) recall

$$
p H^{*}\left(X _ { p - 1 , q } ( C ( r - 1 ) ) \cong \left\{\begin{array}{l}
\mathbb{C} \mathbb{A} \otimes S(A)^{p-1}\{V\} \quad \text { if } q=0 \\
\mathbb{C} \mathbb{A} \otimes S(A)^{p-1} v^{q} \text { if } 0<q<p-1 .
\end{array}\right.\right.
$$

This summand induced from the following transfer. Recall $[a, c]=b$ in $G(r, e)$ and $c^{*}\left(y_{2}\right)=y_{2}+y_{1}$ in $H^{*}\left(\left\langle a, b, c^{p}\right\rangle\right)=H^{*}(C(r-1))$. Hence we can compute

$$
\left.\operatorname{Tr}_{C(r-1)}^{G}\left(y_{2}^{p-1}\right)\right|_{C(r-1)}=\sum_{j}\left(y_{2}-j y_{1}\right)^{p-1}=-y_{1}^{p-1}
$$

which implies that $\operatorname{Tr}_{C(r-1)}^{G}\left(y_{2}^{p-1}\right)=-y_{1}^{p-1}$ since $H^{*}(P) \subset H^{*}(C(r-$ $1))$. Define $\Phi \in A(P, P)$ by

$$
\Phi: P>C(r-1) \stackrel{a \leftrightarrow b}{\rightarrow} C(r-1) \subset P .
$$

Then we have

$$
\Phi\left(v^{q} y_{1}^{p-1}\right)=\operatorname{Tr}_{C(r-1)}^{P}\left(v^{q} y_{2}^{p-1}\right)=-v^{q} y_{1}^{p-1} .
$$

Hence $\Phi\left(H^{*}\left(Y_{p-1, q}(E)\right)\right) \cong \mathbb{C} \mathbb{A}\left\{y_{1}^{p-1} v^{q}\right\} \cong H^{*}\left(Y_{p-1, q}(E)\right)$. Thus we have

$$
Y_{i, q} \cong\left\{\begin{array}{l}
X_{0,0} \vee\left(X_{p-1,0}(C(r-1)) \vee \vee_{j=e v}^{p-3} L(1, j) \quad \text { if } i=q=0\right. \\
X_{0, q} \vee X_{p-1, q}(C(r-1)) \quad \text { if } i=0, q \neq 0 \\
X_{1,0} \vee \vee_{j=o d d} L(1, j) \quad \text { if } i=1, q=0 \\
X_{1, q} \text { if } i=1, q \neq 0 .
\end{array}\right.
$$


Theorem 7.4. For $r>4$, we have

$$
\begin{gathered}
H^{*}\left(X _ { i , q } ( G ( r , e ) ) \cong \left\{\begin{array}{l}
H^{*}\left(\left(\vee_{j=e v}^{p-3} X_{j, 0}(C(r-1))\right) \vee L(1,0)\right) \quad \text { if } i=q=0 \\
H^{*}\left(\vee_{j=e v}^{p-3} X_{j, q}(C(r-1))\right) \text { if } i=0, q \neq 0 \\
H^{*}\left(\vee_{j=o d d}^{p-2} X_{j, q}(C(r-1)) \text { if } i=1\right.
\end{array}\right.\right. \\
\cong\left\{\begin{array}{l}
\mathbb{C} \mathbb{A} \otimes\left(k\{1\} \oplus \oplus_{j=e v>0}^{p-3} k\left\{y_{1}^{j} V, w_{-j} v^{j}\right\}\right) \text { if } i=q=0 \\
\mathbb{C} \mathbb{A} \otimes\left(\oplus_{j=e v}^{p-3} k\left\{y_{1}^{j} v^{q}, w_{-j} j v^{j+q}\right\}\right) \text { if } i=0, q \neq 0 \\
\mathbb{C} \mathbb{A} \otimes\left(\oplus_{j=o d d}^{p-2} k\left\{y_{1}^{j} V, w_{-j} v^{j}\right\}\right) \text { if } i=1, q=0 \\
\mathbb{C} \mathbb{A} \otimes\left(\oplus_{j=o d d}^{p-2} k\left\{y_{1}^{j} v^{q}, w_{-j} v^{j+q}\right\}\right) \text { if } i=1, q \neq 0 .
\end{array}\right.
\end{gathered}
$$

where $w_{-j}=w_{p-1-j}$.

Proof. We will prove the case $i=q=0$, and other cases are proved similarly. From the above isomorphism for $Y_{i, q}$, we have

$$
H^{*}\left(X_{0,0}\right) \cong H^{*}\left(Y_{0,0}\right) \ominus H^{*}\left(X_{p-1,0}(C(r-1))\right) \ominus \oplus_{j=e v}^{p-3} H^{*}(L(1, j)) .
$$

Using Lemma 7.3 , we see $H^{*}\left(Y_{0,0}\right) \cong \oplus_{j: e v}^{p-1} H^{*}\left(Y_{j, 0}(C(r-1))\right.$. Hence we can write $H^{*}\left(X_{0,0}\right) \cong A \oplus B$ with

$$
\begin{aligned}
& A=\oplus_{j=e v}^{p-3} H^{*}\left(Y_{j, 0}(C(r-1)) \ominus \oplus_{j=e v}^{p-3} H^{*}(L(1, j)),\right. \\
& B=H^{*}\left(Y _ { p - 1 , 0 } ( C ( r - 1 ) ) \ominus H ^ { * } \left(X_{p-1,0}(C(r-1)) .\right.\right.
\end{aligned}
$$

Here we have

$$
A \cong \oplus_{j=e v}^{p-3} H^{*}\left(X_{j, 0}\right)(C(r-1)) \quad \text { and } \quad B \cong H^{*}(L(1,0))
$$

Thus we have the first isomorphism in the theorem for $i=q=0$.

The second isomorphism follows from

$$
H^{*}\left(X_{0,0}\right) \cong A \oplus H^{*}(L(1,0)) \cong \mathbb{C} \mathbb{A}\{1\} \oplus \oplus_{0<j=e v}^{p-3} H^{*}\left(X_{j, 0}(C(r-1)) .\right.
$$

Here we used $H^{*}\left(X_{0,0}(C(r-1))\right) \oplus H^{*}(L(1,0)) \cong \mathbb{C} \mathbb{A}\{1\}$.

(II) $r=4$.

In this case cohomology is the same as (I). However the stable splitting is not same as $(I)$ and it is also given by Dietz and Priddy Di-Pr].

$$
\begin{aligned}
B G(r, e) \cong \vee_{i, q} X_{i, q}(G(r, e)) & \vee \vee_{q} X_{p-1, q}(C(r-1)) \\
\vee_{q} L(2, q) & \vee \vee_{q} L(1, q)
\end{aligned}
$$

where $i \in \mathbb{Z} / 2$ and $0 \leq q \leq p-2$.

The problems are only to see that these $H^{*}(L(2, q))$ go to what $H^{*}\left(Y_{i, q^{\prime}}\right)$. Let us consider $\Phi \in A(P, P)$ such that

$$
\Phi: P>E>\left\langle a, c^{p}\right\rangle \stackrel{a \leftrightarrow c^{p}}{\rightarrow}\left\langle a, c^{p}\right\rangle \subset P .
$$

Then we can compute (for $d_{2}=y_{1} v$ )

$$
\Phi\left(d_{2}^{q} y_{1}^{p-1}\right)=\operatorname{Tr}_{E}^{P} \operatorname{Tr}_{\left\langle a, c^{p}\right\rangle}^{E}\left(d_{2}^{q} u^{p-1}\right)=\operatorname{Tr}_{E}^{P}\left(d_{2}^{q}\left(y_{2}^{p-1}-C\right)\right)
$$




$$
=\left.\operatorname{Tr}_{E}^{P}\left(d_{2}^{q}\left(y_{2}^{p-1}-C\right)\right)\right|_{E}=-d_{2}^{q} y_{1}^{p-1}
$$

from (3) in $\S 6$ and the arguments before Lemma 7.4. This means $y_{1}^{q+p-1} v^{k}$ is in the image from $H^{*}(L(2, q)) \subset H^{*}\left(\left\langle a, c^{p}\right\rangle\right)$. Note if $q$ is even, then $y_{1}^{k+p-1} v^{q} \in H^{*}\left(Y_{0, q}\right)$, otherwise it is in $H^{*}\left(Y_{1, q}\right)$. Hence we see

$$
\operatorname{Tr}_{\left\langle a, c^{p}\right\rangle}^{P} H^{*}(L(2, q)) \subset \begin{cases}H^{*}\left(Y_{0, q}\right) & q=\text { even } \\ H^{*}\left(Y_{1, q}\right) & q=\text { odd } .\end{cases}
$$

In particular, note that

$$
D_{2} y_{1}^{p-1} \in H^{*}\left(Y_{p-1,0}(E)\right) \subset H^{*}\left(Y_{0,0}(P)\right)
$$

is in the image from $H^{*}(L(2,0))$. However note $D_{2} C \in H^{*}\left(Y_{0,0}(E)\right) \subset$ $H^{*}\left(Y_{0,0}(P)\right)$ is not in the image from $H^{*}(L(2,0))$. while it is so in $H^{*}\left(Y_{0,0}(E)\right)$.

Thus we have

$$
Y_{i, q} \cong\left\{\begin{array}{l}
X_{0,0} \vee\left(X_{p-1,0}(E) \vee L(2,0) \vee L(1,0)\right) \vee \vee_{j=e v>0}^{p-3} L(1, j) \\
\quad \text { if } i=q=0 \\
X_{0, q} \vee X_{p-1, q}(E) \vee L(2, q) \quad \text { if } i=0, q=e v \neq 0 \\
X_{0, q} \vee X_{p-1, q}(E) \text { if } i=0, q=\text { odd } \neq 0 \\
X_{1,0} \vee \vee_{j=o d d} L(1, j) \quad \text { if } i=1, q=0 \\
X_{1, q} \text { if } i=1, q=e v \neq 0 . \\
X_{1, q} \vee L(2, q) \text { if } i=1, q=\text { odd } \neq 0 .
\end{array}\right.
$$

Then we have

Theorem 7.5. For $P=G(4, e)$, the cohomology $H^{*}\left(X_{i, q}\right)$ is isomorphic to

$$
\cong\left\{\begin{aligned}
& \mathbb{C} \mathbb{A} \otimes\left(k\{1\} \oplus \oplus_{0<j=e v}^{p-3} k\left\{y_{1}^{j} V, w_{-j} v^{j}\right\}\right) \quad \text { if } i=q=0 \\
& \mathbb{C} \mathbb{A} \otimes\left(\oplus_{0<j=e v \neq q}^{p-3} k\left\{y_{1}^{j} v^{q}, w_{-j} v^{j+q}\right\}\right) \\
& \oplus \mathbb{D} \mathbb{A}\left\{y_{1}^{q} v^{q}\right\} \oplus \mathbb{C} \mathbb{A}\left\{w_{-q} v^{2 q}\right\} \quad \text { if } i=0, q=\text { even } \neq 0 \\
& \mathbb{C} \mathbb{A} \otimes\left(\oplus_{j=e v}^{p-3} k\left\{y_{1}^{j} v^{q}, w_{-j} j v^{j+q}\right\}\right) \quad \text { if } i=0, q=\text { odd } \neq 0 \\
& \mathbb{C} \mathbb{A} \otimes\left(\oplus_{j=\text { odd }}^{p-2} k\left\{y_{1}^{j} V, w_{-j} v^{j}\right\}\right) \quad \text { if } i=1, q=0 \\
& \mathbb{C} \mathbb{A} \otimes\left(\oplus_{j=\text { odd }}^{p-2} k\left\{y_{1}^{j} v^{q}, w_{-j} v^{j+q}\right\}\right) \quad \text { if } i=1, q=\text { even } \neq 0 \\
& \mathbb{C} \mathbb{A} \otimes\left(\oplus_{j=\text { odd } \neq q}^{p-3} k\left\{y_{1}^{j} v^{q}, w_{-j} v^{j+q}\right\}\right) \\
& \oplus \mathbb{D} \mathbb{A}\left\{y_{1}^{q} v^{q}\right\} \oplus \mathbb{C} \mathbb{A}\left\{w_{-q} v^{2 q}\right\} \quad \text { if } i=1, q=\text { odd } \neq 0 .
\end{aligned}\right.
$$

Proof. When $i=q=0$, the isomorphism follows from

$$
H^{*}\left(X_{0,0}(G(4, e)) \cong H^{*}\left(X_{0,0}(G(r, e))\right) \quad \text { for } r>4\right. \text {. }
$$


This fact is shown from the decomposition $Y_{0,0}$ above and

$$
H^{*}\left(X_{p-1,0}(C(r-1)) \cong H^{*}\left(X_{p-1,0}(C(3)) \vee L(2,0)\right) \quad \text { for } r>4 .\right.
$$

When $i=0, q=e v \neq 0$, the fact

$$
H^{*}\left(X _ { 0 , q } ( G ( 4 , e ) ) \cong H ^ { * } \left(X_{0, q}\left(G\left(r, e^{\prime}\right)\right) \ominus H^{*}(L(2, q))\right.\right.
$$

implies the isomorphism in the theorem. The other cases are also seen similarly.

\section{Relations among $B P$ With $\operatorname{rank}_{p} P=2$.}

In this section, we see Theorem 1.1 in the introduction. For a group $G$ with $\operatorname{rank}_{p} G=2$, let us write by $X_{i, q}(G)$ (or $X_{i}(G)$ for a metacyclic group) the corresponding irreducible stable homotopy summand.

Recall that a non-dominant summand $X$ is the irreducible summand corresponding to an $A(G, G)$-simple module $S(G, Q, V)$ for a proper subgroup $Q$ and a simple Out $(Q)$-module $V$. From Dietz-Priddy, the following lemma is immediate.

Lemma 8.1. Let $G=C(r)$ (or $G(r+1, e))$ for $r \geq 3$. Then for $0 \leq$ $q \leq p-2$, a non-dominant summand is $L(1, q), L(2, q)\left(\right.$ or $X_{p-1, q}(C(r))$ for $G=G(r+1, e))$.

Let us use the notation such that for stable homotopy spaces $A, B$, the notation $A \cong{ }_{H} B$ means $H^{*}(A) \cong H^{*}(B)$ as graded modules. Theorem 1.1 in the introduction is a immediate consequence of the above lemma and the following theorem about dominant summands.

Theorem 8.2. Let $G=C(r)$ (or $G(r+1, e)$ ) for $r \geq 3$. Given $0 \leq i \leq p-1$ (or $i=0$ or 1 ) and $0 \leq q \leq p-2$, there are $a_{j}, b_{k}, c$ which are 0 or 1 such that we have the isomorphism

$$
X_{i, q}(G) \cong_{H} \vee_{j=0}^{p-1} a_{j} X_{j, q}(E) \vee \vee_{k=0}^{p-2} b_{k} L(2, k) \vee c L(1,0)
$$

In particular, $c=1$ if and only if $i=q=0$ and $G=G(r+1, e)$.

Proof. We first consider $G=C(r)$, for $r>3$. Since $C(r) \cong_{H} E$, we see $Y_{i, q}(C(r)) \cong_{H} Y_{i, q}(E)$. Let $q=0$. Then from the formula for $Y_{i, q}$ just before Lemma $6.3, Y_{i, 0}(C(r)) \cong X_{i, 0}(C(r)) \vee L(1, i)$.

On the other hand, from Corollary 6.8, we see

$$
Y_{i, 0}(E) \cong_{H} X_{i, 0}(E) \vee L(1, i) \vee b L(2,-i) \quad \text { for } b=0 \text { or } 1 .
$$

Hence $X_{i, 0}(C(r)) \cong_{H} X_{i, 0}(E) \vee b L(2,-i)$, and $(*)$ is satisfied in this case, in particular note $c=0$. The case $q \neq 0$ is shown similarly by using Corollary 6.8

$$
Y_{i, q}(C(r)) \cong_{H} X_{i, q}(E) \vee b^{\prime} L(2, i) \vee b^{\prime \prime} L(2,-i) \quad b^{\prime}, b^{\prime \prime}=0 \text { or } 1 .
$$


The case $G=G(r+1, e), r>3$ is immediate from the first isomorphism in Theorem 7.4 and the result for $C(r)$. We also note $c=1$ if and only if $i=q=0$. Hence we can write

$$
X_{i, q}(G(r+1, e)) \cong_{H} \vee \vee_{j=0}^{p-2} a_{j} X_{j, q}(E) \vee \vee_{k=0}^{p-2} b_{k} L(2, k) \vee c L(1,0)
$$

where $0 \leq a_{j}, b_{k}, c \leq 1$.

The fact $0 \leq b_{k} \leq 1$ is shown by the following arguments. Note if $Y_{j, q}(E)$ contains $b^{\prime} L(2, k)$, then $b^{\prime}=1$ and $k=j$ or $k=-j$ from Corollary 6.8. Therefore $\vee_{j=0}^{p-2} Y_{j, q}(E)$ contains $b L(2, k)$ for $b \leq 2$. Suppose $b_{k}=2$ and $k \neq 0$. Since $Y_{j, q}(E)$ contains $L(2, j)$ only when $j=q$, we can assume $k=q$. However $Y_{p-1-q, q}(E)$ does not contain $L(2, q)$ from Corollary 6.8. Hence $b_{k} \neq 2$.

Let $G=G(4, e)$. First note that $Y_{i, q}(G(r+1, e)) \cong_{H} Y_{i, q}(G(4, e))$. When $i=q=0$, the isomorphism $(*)$ is immediate from $X_{0,0}(G(4, e)) \cong_{H}$ $X_{0,0}(G(r+1, e))$. When $i=0, q=e v \neq 0$, we recall

$$
H^{*}\left(X _ { 0 , q } ( G ( 4 , e ) ) \cong H ^ { * } \left(X_{0, q}(G(r+1, e)) \ominus H^{*}(L(2, q))\right.\right.
$$

Here $X_{0, q}(G(r+1, e)) \cong_{H} \bigvee_{j=e v e n}^{p-3} X_{j, q}(C(r))$. The last space contains

$$
X_{q, q}(C(r)) \cong_{H} X_{q, q}(E) \vee L(2, q) \vee b L(2,2 q) \quad \text { for } b=0 \text {, or } 1
$$

from Corollary 6.8, which implies the isomorphism in the theorem. The other cases are also seen similarly.

Example. Let $p=7$ and $r>3$. We see from Corollary 6.8

$$
X_{5,2}(C(r)) \cong Y_{5,2}(C(r)) \cong_{H} Y_{5,2}(E) \cong X_{5,2}(E) \vee L(2,1)
$$

We have $H^{*}\left(Y_{5,2}(E)\right) \cong \mathbb{C} \mathbb{A}\left\{y_{1}^{5} v^{2}, w_{1} v\right\}$ and $H^{*}(L(2,1)) \cong \mathbb{C} \mathbb{B}\left\{u^{p-1} d_{2}^{1}\right\}$ which maps to $w_{1} v$ by the transfer. Hence

$$
H^{*}\left(X_{5,2}(E)\right) \cong H^{*}\left(Y_{5,2}(E)\right) \ominus H^{*}\left(L(2,1) \cong \mathbb{C} \mathbb{A}\left\{y_{1}^{5} v^{2}\right\} \oplus \mathbb{D} \mathbb{A}\left\{w_{1} v\right\}\right.
$$

by using $\mathbb{C} A \ominus \mathbb{C B}\{C\} \cong \mathbb{D} \mathbb{A}$. For the case $X_{6,0}(E)$, we have $H^{*}\left(Y_{6,0}(E)\right) \cong$ $\left.H^{*}\left(X_{6,0}(E)\right) \oplus H^{*}(L(2,0)) \vee L(1,0)\right)$. Hence we see also

$$
H^{*}\left(X_{6,0}(E)\right) \cong \mathbb{C} \mathbb{A}\{Y\} \ominus \mathbb{C B}\{Y\} \cong \mathbb{D} \mathbb{A}\{V Y\} .
$$

(See also the example after Corollary 6.8.)

Example. We consider the case $p=7$ and $q=2$. The cohomology $H^{*}\left(Y_{j, 2}\right) \cong \mathbb{C} \mathbb{A}\left\{y_{1}^{j} v^{2}, w_{-j} v^{j+2}\right\}$. Therefore for $r^{\prime}>4$, we see

$$
\begin{gathered}
H^{*}\left(X_{0,2}\left(G\left(r^{\prime}, e\right)\right)\right) \cong H^{*}\left(\vee_{j=0,2,4} Y_{j, 2}\right) \cong \mathbb{C} \mathbb{A}\left\{v^{2}, y_{1}^{2} v^{2}, y_{1}^{4} v^{2}, w_{4} v^{4}, w_{2}\right\} \\
H^{*}\left(X_{0,2}(G(4, e))\right) \cong H^{*}\left(\vee_{j=0,2,4} Y_{j, 2}\right) \ominus H^{*}(L(2,2)) \\
\cong \mathbb{C} \mathbb{A}\left\{v^{2}, y_{1}^{4} v^{2}, w_{2}, w_{4} v^{4}\right\} \oplus \mathbb{D} \mathbb{A}\left\{y_{1}^{2} v^{2}\right\}
\end{gathered}
$$


Next, we study split metacyclic groups, For stable spaces $X=$ $X_{i, j}(G)$, let $S X$ be the virtual object defined by

$$
H^{*}(S X)=H^{*}(X) \cap \mathbb{C} \mathbb{A} \otimes\left(\oplus_{q=0}^{p-2} k\left\{1, y_{1}, \ldots, y_{1}^{p-2}\right\}\left\{v^{q}\right\}\right)
$$

where we identify it as the submodule of $\mathbb{C} \mathbb{A} \otimes\left(\oplus_{q} S(A)^{*}\left\{v^{q}\right\}\right) \subset H^{*}(E)$ in Theorem 6.1. Then we see

$$
H^{*}(S(B E)) \cong \mathbb{C} \mathbb{A} \otimes\left(\oplus_{q}\left(k\left\{1, y_{1}, \ldots, y^{p-2}\right\}\left\{v^{q}\right\}\right) \cong k[y, v]\right.
$$

identifying $C=Y=y_{1}^{p-1}$ as graded modules.

Recall that $H^{*}(M(\ell, m, n)) \cong k[y, v]$ with $|v|=2 p^{m-\ell}$. Let us write $M=(m-1, m, n))$ so that

$$
H^{*}\left(Y_{q}(M)\right) \cong \oplus_{j=0}^{p-2} H^{*}\left(S Y_{j, q}(E)\right)
$$

The results in $\S 5$ imply the following theorem

Theorem 8.3. Let $M=M(m-1, m, n)$ and $r>3$. Then we have

$$
H^{*}\left(X_{q}(M)\right) \cong\left\{\begin{array}{l}
\oplus_{j}^{p-2} H^{*}\left(S X_{j, q}(C(r))\right) \quad \text { if }(m, n) \neq(2,1) \\
\oplus_{j}^{p-2} H^{*}\left(S X_{j, q}(E)\right) \quad \text { if }(m, n)=(2,1) .
\end{array}\right.
$$

Proof. The case $q \neq 0$ is shown from

$$
H^{*}\left(Y_{q}(M)\right) \cong H^{*}\left(X_{q}(M)\right) \cong H^{*}\left(X_{q}(M(1,2,1)) \vee L(2, q)\right) .
$$

The case $q=0$ is also proved similarly.

At last in this section, we consider the cases $m-\ell>1$. From the results in $\S 5$, it is almost immediate

Proposition 8.4. Let $m-\ell>1$. Then we have

$$
H^{*}\left(X _ { i } ( M ( \ell , m , n ) ) \cong H ^ { * } \left(X_{i}(M(m-1, m, n)) \cap k\left[y, v^{p^{m-\ell-1}}\right] .\right.\right.
$$

From these results, we get

Theorem 8.5. For $p \geq 5$, let $P$ be a non-abelian $p$-group of $\operatorname{rank}_{p} P=$ 2 , and $X_{i}(P)$ be an irreducible component of $B P$. Then there are graded submodules $H^{*}(P, j) \subset H^{*}\left(X_{j}\left(p_{+}^{1+2}\right)\right)$ such that

$$
H^{*}\left(X_{i}(P)\right) \cong \oplus_{j \in J(i, P)} H^{*}(P, j)
$$

for some index set $J(i, P)$. When $P$ is not a metacyclic group, we can take $H^{*}(P, j)=H^{*}\left(X_{j}\left(p_{+}^{1+2}\right)\right)$.

Example. Let $p=7, q=2$. Then we have

$$
\oplus_{j=1}^{5} H^{*}\left(S Y_{j, 2}(E)\right) \cong \mathbb{C} \mathbb{A}\left\{1, y, \ldots, y^{5}\right\}\left\{v^{2}\right\} .
$$

Hence we have

$$
X_{2}(M(1,2,1)) \cong \oplus_{j}^{5} H^{*}\left(S Y_{i, 2}(E)\right) \ominus H^{*}(S(L(2,2) \vee L(2,4)))
$$




$$
\cong \mathbb{C} \mathbb{A}\left\{1, y_{1}, y_{1}^{3}, y_{1}^{4}, y_{1}^{5}\right\}\left\{v^{2}\right\} \oplus \mathbb{D} \mathbb{A}\left\{y_{1}^{2} v^{2}\right\}
$$

which is still given in Theorem 5.3 (letting $S(L(2,2) \vee L(2,4)$ ) = $L(2,2))$.

\section{NILPOTENT ELEMENTS}

Let us write $H^{e v e n}(X ; \mathbb{Z}) / p$ by simply $H^{e v}(X)$ so that

$$
H^{e v}(G)=H^{*}(G) \oplus N(G)
$$

where $N(G)$ is the nilpotent ideal in $H^{e v}(G)$.

At first, we consider metacyclic groups. Since $B P$ is irreducible in non split cases, we only consider in split cases. Recall

$$
P=M(\ell, m, n)=\left\langle a, b \mid a^{p^{m}}=b^{p^{n}}=1,[a, b]=a^{p^{\ell}}\right\rangle
$$

for $m>\ell \geq \max (m-n, 1)$.

(I) Split metacyclic groups with $\ell>m-n$.

By Diethelm [Dim], its mod p-cohomology is

$$
H^{*}(P ; \mathbb{Z} / p) \cong k[y, u] \otimes \Lambda(x, z) \quad|y|=|u|=2,|x|=|z|=1 .
$$

Of course all elements in $H^{*}(P ; \mathbb{Z})$ are (higher) $p$-torsion. The additive structure of $H^{*}(P ; \mathbb{Z}) / p$ is decided by that of $H^{*}(P ; \mathbb{Z} / p)$ by the universal coefficient theorem. Hence we have additively (but not as rings)

$$
\begin{aligned}
& H^{*}(P ; \mathbb{Z}) / p \cong H^{*}(\mathbb{Z} / p \times \mathbb{Z} / p ; \mathbb{Z}) \\
\cong & k[y, u]\{1, \beta(x z)=y z-u x\}
\end{aligned}
$$

The element $u \in H^{2}(P ; \mathbb{Z} / p)$ is reduced [Dim] from the spectral sequence

$$
E_{2}^{*, *^{\prime}} \cong H^{*}\left(P /\langle a\rangle ; H^{*^{\prime}}(\langle a\rangle ; \mathbb{Z} / p)\right) \Longrightarrow H^{*}(P ; \mathbb{Z} / p) .
$$

In fact $u=\left[u^{\prime}\right] \in E_{\infty}^{0,2}$ identifying $H^{2}(\langle a\rangle ; \mathbb{Z} / p) \cong k\left\{u^{\prime}\right\}$. Hence $\left.u\right|_{\langle a\rangle}=$ $u^{\prime}$. On the other hand, for the element $v=c_{p^{m-\ell}}(\eta)$ defined in $\S 5$, $\left.v\right|_{\langle a\rangle}=\left(u^{\prime}\right)^{p^{m-\ell}}$ because the total Chern class in $H^{*}(P ; \mathbb{Z} / p)$ is

$$
\left.\sum c_{i}(\eta)\right|_{\langle a\rangle}=\left(1+u^{\prime}\right)^{p^{m-\ell}}=1+\left(u^{\prime}\right)^{p^{m-\ell}} .
$$

Therefore we see $v=u^{p^{m-\ell}} \bmod (y, x z)$ in $H^{*}(P ; \mathbb{Z} / p)$.

Since $H^{*}(P)$ is multiplicatively generated by $y$ and $v$ with $|v| \geq 2 p$ from Theorem 5.1, the element $u$ is not integral class (i.e. $u \notin \operatorname{Im}(\rho)$ for $\left.\rho: H^{*}(P ; \mathbb{Z}) \rightarrow H^{*}(P ; \mathbb{Z} / p)\right)$. Therefore $x z$ is an integral class since $\operatorname{dim} H^{2}(P ; \mathbb{Z}) / p=2$ from $(1)$, and $H^{2}(P ; \mathbb{Z} / p) \cong k\{y, u, x z\}$. Moreover we have 
Lemma 9.1. The ring of the integral classes in $H^{*}(P ; \mathbb{Z} / p)$ is given as

$$
H^{e v}(P) \cong k[y, v]\left\{1, x z, x z u, \ldots, x z u^{p^{m-\ell}-2}\right\} \subset H^{*}(P ; \mathbb{Z} / p) .
$$

Proof. Each element $y^{i} u^{j}$ is not nilpotent, since $H^{*}(P ; \mathbb{Z} / p) \cong k[y, u]$. Hence for $1 \leq j<p^{m-\ell}$, each element $y^{i} u^{j}$ is not integral. Let $A=$ $k[y, v]\left\{1, x z, \ldots, x z u^{p^{m-\ell}-2}\right\}$. Then note that

$$
A \cong H^{*}(P ; \mathbb{Z} / p) /\left(\mathbb{Z} / p\left\{y^{i} u^{j}\right\} \mid 1 \leq j<p^{m-\ell}\right) .
$$

Hence $H^{e v}(P) \subset A$ in the lemma.

On the other hand $\operatorname{dim} A=n+1$ when the degree is $2 n<2 p^{m-\ell}$ which is equal to $\operatorname{dim} H^{e v}(P)$.

Let us write

$$
c_{1}=x z, c_{2}=x z u, \ldots, c_{p^{m-\ell}-1}=x z u^{p^{m-\ell}-2} .
$$

Then $c_{i} c_{j}=(x z)^{2} u^{i+j-2}=0$. Here recall $H^{\text {even }}(P ; \mathbb{Z})$ is multiplicatively generated by $y, c_{i}(\eta)$ by the argument just before Theorem 5.1 (Thomas, Huebuschmann [Th], [Hu]). Hence we know

Lemma 9.2. We have $c_{i}=\lambda_{i} c_{i}(\eta) \bmod \left(y, c_{1}, \ldots, c_{i-1}\right)$ with $\lambda_{i} \neq 0 \in$ $\mathbb{Z} / p$.

Proof. By induction, assume the equation for $i-1$. Since $c_{i}=x z u^{i-1}$, it is not represented by the polynomial of $y, c_{1}, \ldots, c_{i-1}$. So it must be represented by $c_{i}(\eta)$ by the result of Thomas and Huebuschmann.

Thus we get

Theorem 9.3. Let $P$ be a split metacyclic group $M(\ell, m, n)$ with $\ell>m-n$. Then we have

$$
H^{e v}(P) \cong k[y, v]\left\{1, c_{1}, \ldots, c_{p^{m-\ell}-1}\right\} \quad \text { with } c_{i} c_{j}=0
$$

that is $N(P) \cong k[y, v]\left\{c_{1}, \ldots, c_{p^{m-\ell}-1}\right\}$.

As $O u t(P)$ modules, $k\left\{c_{i}\right\}=k\left\{x z u^{i}\right\} \cong S_{j}$ when $i=j \bmod (p-1)$. Therefore we have

Corollary 9.4. Let $P$ be a split metacyclic group $M(\ell, m, n)$ with $\ell>m-n$. Then

$$
H^{e v}\left(X_{i}\right) \cong H^{*}\left(X_{i}\right) \oplus k[y, V]\left\{v^{r} c_{s} \mid r+s=i \bmod (p-1)\right\}
$$

where $1 \leq s \leq p^{m-\ell}-1$. 
(II) Split metacyclic groups $P=M(\ell, m, n)$ with $\ell=m-n$.

By also Diethelm [Dim], its mod $p$-cohomology is

$$
H^{*}(P ; \mathbb{Z} / p) \cong k\left[y, v^{\prime}\right] \otimes \Lambda\left(a_{1}, \ldots, a_{p-1}, b, w\right) /\left(a_{i} a_{j}=a_{i} y=a_{i} w=0\right)
$$

where $\left|a_{i}\right|=2 i-1,|b|=1,|y|=2,|w|=2 p-1,\left|v^{\prime}\right|=2 p$. So we see

$$
H^{*}(P ; \mathbb{Z} / p) / \sqrt{0} \cong k\left[y, v^{\prime}\right] .
$$

Note that additively $H^{*}(P ; \mathbb{Z}) / p \cong H^{*}\left(p_{-}^{1+2} ; \mathbb{Z}\right) / p$, which is well known. In particular, we get additively

$$
\begin{aligned}
& H^{e v}(P) \cong\left(k[y] \oplus k\left\{c_{1}, \ldots, c_{p-1}\right\}\right) \otimes k\left[v^{\prime}\right] \quad\left(\text { with } c_{i}=a_{i} b\right) \\
& \cong\left(k[y] \oplus k\left\{c_{1}, \ldots, c_{p-1}\right\}\right) \otimes k[v]\left\{1, v^{\prime}, \ldots,\left(v^{\prime}\right)^{p^{m-\ell-1}-1}\right\} .
\end{aligned}
$$

Therefore $H^{e v}(P)$ is additively isomorphic to

$$
H^{e v}(P) \cong \oplus_{i, j} k[v]\left\{a_{i} b\left(v^{\prime}\right)^{j}\right\} \oplus \oplus_{j} k[v, y]\left\{\left(v^{\prime}\right)^{j}\right\}
$$

where $1 \leq i \leq p-1$ and $0 \leq j \leq p^{m-\ell-1}-1$. Here $a_{i} b\left(v^{\prime}\right)^{j}$ is nilpotent and hence integral class and let $c_{j p+i}=a_{i} b\left(v^{\prime}\right)^{j}$. The element $\left(v^{\prime}\right)$ is not nilpotent and we can take as the integral class $w b$ of dimension $2 p$. Let us write $c_{p j}=w b\left(v^{\prime}\right)^{j-1}$. Thus we have

Theorem 9.5. Let $P$ be a split metacyclic group $M(\ell, m, n)$ with $\ell=m-n$. Then

$$
H^{e v}(P) \cong k[y, v] \oplus k[y, v]\left\{c_{i} \mid i=0 \bmod (p)\right\} \oplus k[v]\left\{c_{i} \mid i \neq 0 \bmod (p)\right\}
$$

where $i$ ranges $1 \leq i \leq p^{m-\ell}-1$. Here the multiplications are given by $c_{i} c_{j}=0$ for $0<i, j<p^{m-\ell}$ and $y c_{k}=0$ for $k \neq 0 \bmod (p)$.

Hence we have

Corollary 9.6. Let $P=M(\ell, m, n)$ for $\ell=m-n$. Then

$$
\begin{gathered}
H^{e v}\left(X_{i}\right) \cong H^{*}\left(X_{i}\right) \oplus k[y, V]\left\{v^{r} c_{s} \mid s=0 \bmod (p), r+s=i \bmod (p-1)\right\} \\
\oplus k[V]\left\{v^{r} c_{s} \mid s \neq 0 \bmod (p), r+s=i \bmod (p-1)\right\} .
\end{gathered}
$$

(III) groups $P=C(r)$ or $G\left(r^{\prime}, e\right)$.

Let $P=C(r)$. Then it is known ([Ya1])

$$
N(P)= \begin{cases}k[v]\left\{c_{2}, \ldots, c_{p-2}\right\} & r=3, \quad\left|c_{i}\right|=2 i \\ k[v]\left\{c_{1}, \ldots, c_{p-2}\right\} & r \geq 4\end{cases}
$$

Each $c_{i}$ is defined as a Chern class and as $O u t(C(r))$ modules, we see $k\left\{c_{i}\right\} \cong \operatorname{det}^{i}$.

For $P=G(r, e)$, each $c_{i}$ is invariant under the action $c^{*}$. Hence we have

$$
N(G(r+1, e)) \cong N(C(r)) .
$$


Theorem 9.7. Let $P=C(r)$ or $G(r+1, e)$ for $r \geq 3$. Then

$$
\begin{aligned}
& H^{e v}\left(X_{j, i}\right) \cong\left\{\begin{array}{l}
H^{*}\left(X_{0, i}\right) \oplus k[V]\left\{v^{r} c_{s} \mid r+s=i \bmod (p-1)\right\} \quad j=0 \\
H^{*}\left(X_{j, i}\right) \quad j \neq 0 .
\end{array}\right. \\
& \text { where s ranges }\left\{\begin{array}{l}
2 \leq s \leq p-2 \text { for } r=3, \\
1 \leq s \leq p-2 \quad \text { for } r \geq 4 .
\end{array}\right.
\end{aligned}
$$

\section{Chow Rings And motives}

For a smooth quasi projective algebraic variety $X$ over $\mathbb{C}$, let $C H^{*}(X)$ be the Chow ring generated by algebraic cycles of codimension $*$ modulo rational equivalence. There is a natural (cycle) map

$$
c l: C H^{*}(X) \rightarrow H^{2 *}(X(\mathbb{C}) ; \mathbb{Z}) .
$$

where $X(\mathbb{C})$ is the complex manifold of $\mathbb{C}$-rational points of $X$.

Let $V_{n}$ be a $G$ - $\mathbb{C}$-vector space such that $G$ acts freely on $V_{n}-S_{n}$, with $\operatorname{codim}_{V_{n}} S_{n}=n$. Then it is known that $\left(V_{n}-S_{n}\right) / G$ is a smooth quasiprojective algebraic variety. Then it is known that $C H^{*}\left(\left(V_{n}-S_{n}\right) / G\right)$ is independent of the choice of $V_{n}, S_{n}$. Hence Totaro defines the Chow ring of $B G([\mathrm{To} 1])$ by

$$
C H^{*}(B G)=\lim _{n \rightarrow \infty} C H^{*}\left(\left(V_{n}-S_{n}\right) / G\right) .
$$

Moreover we can approximate $\mathbb{P}^{\infty} \times B G$ by smooth projective varieties from Godeaux-Serre arguments ([To1]).

Let $P$ be a $p-$ group. By the Segal conjecture, the $p$-complete automorphism $\{B P, B P\}$ of stable homotopy groups is isomorphic to $A(P, P)_{\mathbb{Z}_{p}}$, which is generated by transfers and map induced from homomorphisms. Since $C H^{*}(B P)$ also has the transfer map, we see $C H^{*}(B P)$ is an $A(P, P)$-module. For an $A(P, P)$-simple module $S$, recall $e_{S}$ is the corresponding idempotent element and $X_{S}=e_{S} B P$ the irreducible stable homotopy summand. Let us define

$$
C H^{*}\left(X_{S}\right)=e_{S} C H^{*}(B P)
$$

so that the following diagram commutes

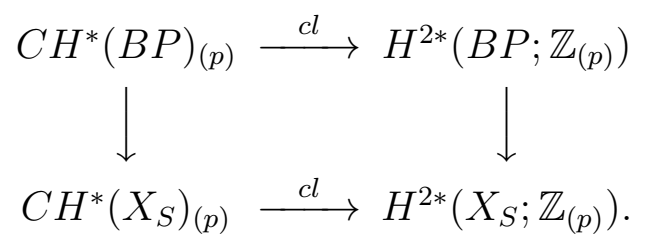

For smooth schemes $X . Y$ over a field $K$, let $\operatorname{Cor}(X, Y)$ be the group of finite correspondences from $X$ to $Y$ (which is a $\mathbb{Z}_{p}$-module on the set of closed subvarieties of $X \times_{K} Y$ which are finite and surjective 
over some connected component of $X$. Let $\operatorname{Cor}\left(K, \mathbb{Z}_{p}\right)$ be the category of smooth schemes whose groups of morphisms $\operatorname{Hom}(X, Y)=$ $\operatorname{Cor}(X, Y)$.Voevodsky constructs the triangulated category $D M=$ $D M\left(K, \mathbb{Z}_{p}\right)$ which contains the category $\operatorname{Cor}\left(K, \mathbb{Z}_{p}\right)$ (and limit of objects in $\left.\operatorname{Cor}\left(K, \mathbb{Z}_{p}\right)\right)$.

Lemma 10.1. Let $S$ be a simple $A(P, P)$-module. Then there is a motive $M_{S} \in D M\left(\mathbb{C}, \mathbb{Z}_{p}\right)$ such that

$$
C H^{*}\left(M_{S}\right) \cong C H^{*}\left(X_{S}\right)=e_{S} C H^{*}(B P) .
$$

Proof. Let $P$ act freely on $V-S$ so that $(V-S) / G$ approximates $B G$. For a subgroup $i: H \subset P$, the induced map $i^{*}$ is defined from the projection

$$
p r:(V-S) / H \rightarrow(V-S) / G \text {. }
$$

This corresponds an element in morphism in $\operatorname{Cor}\left(\mathbb{C}, \mathbb{Z}_{p}\right)$

$$
\operatorname{pr}^{*}=\{(x, p r(x)) \mid x \in(V-S) / H\} \in \operatorname{Cor}((V-S) / H,(V-S) / G) .
$$

The transfer map is induced from

$$
\operatorname{tr}=\{(\operatorname{pr}(x), x) \mid x \in(V-S) / H\} \in \operatorname{Cor}((V-S) / G,(V-S) / H)
$$

also by the definition of (finite) correspondences. Therefore each element in $A(P, P)$ is represented by a morphism of the category $D M=$ $D M\left(\mathbb{C}, \mathbb{Z}_{p}\right)$. Moreover $D M$ is a triangulated category and $\operatorname{Im}\left(e_{S}\right)$ (i.e. the cone of $\left.e_{S}\right)$ is an object of $D M$.

Remark. Of course $M_{S}$ is (in general) not irreducible, while $X_{S}$ is irreducible.

The category $C h o w^{e f f}\left(K, \mathbb{Z}_{p}\right)$ of (effective) pure Chow motives is defined as follows. An object is a pair $(X, p)$ where $X$ is a projective smooth variety over $K$ and $p$ is a projector, i.e. $p \in \operatorname{Mor}(X, X)$ with $p^{2}=p$. Here a morphism $f \in \operatorname{Mor}(X, Y)$ is defined as an element $f \in C H^{\operatorname{dim}(Y)}(X \times Y)_{\mathbb{Z}_{p}}$. We say that each $M=(X, p)$ is a (pure) motive and define the Chow ring $C H^{*}(M)=p^{*} C H^{*}(X)$, which is a direct summand of $C H^{*}(X)$. We identify that the motive $M(X)$ of $X$ means $(X, i d$.$) . (The category D M\left(K, \mathbb{Z}_{p}\right)$ contains the category $\operatorname{Chow}^{\text {eff }}\left(K, \mathbb{Z}_{p}\right)$.)

It is known that we can approximate $\mathbb{P}^{\infty} \times B P$ by smooth projective varieties from Godeaux-Serre arguments ([To1]). Hence we can get the following lemma since

$$
C H^{*}\left(X \times \mathbb{P}^{\infty}\right) \cong C H^{*}(X)[y] \quad|y|=1 .
$$


Lemma 10.2. Let $S$ be a simple $A(P, P)$-module. There are pure motives $M_{S}(i) \in C h o w^{\text {eff }}\left(\mathbb{C}, \mathbb{Z}_{p}\right)$ such that

$$
\lim _{i \rightarrow \infty} C H^{*}\left(M_{S}(i)\right) \cong C H^{*}\left(X_{S}\right)[y], \quad \operatorname{deg}(y)=1 .
$$

The following theorem is proved by Totaro, with the assumption $p \geq 5$ but without the assumption of transferred Euler classes (since it holds when $p \geq 5$ ).

Theorem 10.3. (Theorem 14.3 in [To2]) Suppose rank $P \leq 2$ and $P$ has a faithful complex representation of the form $W \oplus X$ where $\operatorname{dim}(W) \leq p$ and $X$ is a sum of 1 -dimensional representations. Moreover $H^{e v}(P)$ is generated by transferred Euler classes. Then we have $C H^{*}(P) / p \cong H^{e v}(P)$.

Proof. (See page 179-180 in [To2].) First note the cycle map is surjective, since $H^{e v}(P)$ is generated by transferred Euler classes. Using the Riemann-Roch without denominators, we can show

$$
C H^{*}(B P) / p \cong H^{2 *}(P ; \mathbb{Z}) / p \quad \text { for } * \leq p .
$$

By the dimensional conditions of representations $W \oplus X$ and Theorem 12.7 in [To], we see the following map

$$
\begin{gathered}
C H^{*}(B P) / p \rightarrow \prod_{V} C H^{*}(B V) \otimes_{\mathbb{Z} / p} C H^{\leq p-1}\left(B C_{P}(V)\right) \\
\rightarrow \prod_{V} H^{*}(V ; \mathbb{Z} / p) \otimes_{\mathbb{Z} / p} H^{\leq 2(p-1)}\left(C_{P}(V) ; \mathbb{Z} / p\right)
\end{gathered}
$$

is also injective. Here $V$ ranges elementary abelian $p$-subgroups of $P$ and $C_{P}(V)$ is the centralizer group of $V$ in $P$. So we see that the cycle map is also injective.

Therefore we have

Corollary 10.4. Let $P$ be $C(r), G\left(r^{\prime}, e\right)$ or split metacyclic groups with $m-\ell=1$. Then $C H^{*}(B P) / p \cong H^{e v}(B G)$.

Totaro computed $C H^{*}(B P) / p$ for split metacyclic groups with $m-$ $\ell=1$ in 13.12 in [To]. When $P$ is the extraspecial $p$-groups of order $p^{3}$, the above result is proved in [Ya3].

Theorem 10.5. Let $P$ be a split metacyclic p-group $M(\ell, m, n)$ with $m-\ell=1, C(r)$ for $p \geq 3$, or $G\left(r^{\prime}, e\right)$ for $p \geq 5$. Then for each simple $A(P, P)$-module $S$, there is a motive $M_{S} \in D M\left(\mathbb{C}, \mathbb{Z}_{p}\right)$ with

$$
C H^{*}\left(M_{S}\right) / p \cong H^{e v}\left(X_{S}\right)=H^{e v e n}\left(X_{S} ; \mathbb{Z}\right) / p .
$$


For a cohomology theory $h^{*}(-)$, define the $h^{*}(-)$-theory topological nilpotence degree $d_{0}\left(h^{*}(B G)\right)$ to be the least nonnegative integer $d$ such that the map

$$
h^{*}(B G) / p \rightarrow \prod_{V: e l . a b .} h^{*}(B V) \otimes h^{\leq d}\left(B C_{G}(V)\right) / p
$$

(where $V$ ranges elementary abelian $p$-subgroups of $G$ ) is injective. Note that $d_{0}\left(H^{*}(B G ; \mathbb{Z})\right) \leq d_{0}\left(H^{*}(B G ; \mathbb{Z} / p)\right)$.

Totarto computed it in the many cases of groups $P$ with $\operatorname{rank}_{p} P=$ 2. In particular, if $P$ is a split metacyclic $p$-group for $p \geq 3$, then $d_{0}\left(H^{*}(P ; \mathbb{Z} / p)\right)=2$ and $d_{0}\left(C H^{*}(B P)\right)=1$ when $m-\ell=1$. Hence $d_{0}\left(H^{*}(P ; \mathbb{Z})\right)=2$ for these split metacyclic groups $P$ (for $p \geq 3$ ).

This fact also is shown directly from Theorem 9.3 and 9.5. Let $P=M(\ell, m, n)$ with $m-\ell=1$. Consider the restriction map

$$
H^{e v}(P) \rightarrow H^{e v}(V) \otimes H^{2}(P ; \mathbb{Z}) / p \quad\left(\text { where } V=\left\langle a^{p^{m-1}}\right\rangle \subset Z(P): \text { center }\right)
$$

induced the product map $V \times P \rightarrow P$. Let $\ell=m-1>m-n$. Then the element is defined in Lemma 9.1, 9.2

$c_{j}=x z u^{j-1} \mapsto \sum_{i} u^{j-i-1} \otimes x z u^{i} \equiv u^{j-1} \otimes c_{1} \neq 0 \in H^{e v}(V) \otimes H^{2}(P ; \mathbb{Z}) / p$.

For $\ell=m-n$, we can see $d_{0}\left(H^{*}(P ; \mathbb{Z})\right)=2$ similarly.

\section{REFERENCES}

[Be-Fe] D. J. Benson and M. Feshbach, Stable splittings of classifying spaces of finite groups, Topology 31 (1992), 157-176.

[Ca] G. Carlsson, Equivariant stable homotopy and Segal's Burnside ring conjecture, Ann. Math. 120 (1984), 189-224.

[Dim] T. Diethelm, The mod $p$ cohomology rings of the nonabelian split metacyclic p-groups. Arch. Math 44, (1985), 29-38. 93-103.

[Di] J. Dietz, Stable splitting of classifying space of metacyclic $p$-groups, $p$ odd. J. Pure and Appied Algebra 90 (1993) 115-136.

[Di-Pr] J. Dietz and S. Priddy, The stable homotopy type of rank two $p$-groups, in: Homotopy theory and its applications, Contemp. Math. 188, Amer. Math. Soc., Providence, RI, (1995), 93-103.

[Gr] D. Green, On the cohomology of the sporadic simple group $J_{4}$, Math. Proc. Camb. Phil. Soc. 113 (1993), 253-266.

[Gr-Le] D. Green and I. Leary, Chern classes and extra special groups. Manuscripta Math. 88 (1995) 73-84.

[Ha-Ku] J. C. Harris and N. J. Kuhn, Stable decompositions of classifying spaces of finite abelian p-groups, Math. Proc. Camb. Phil. Soc. 103 (1988), 427449 .

[Hi-Ya1] A. Hida and N. Yagita, Representation of the double Burnside algebra and cohomology of extraspecial p-group. J. Algebra 409 (2014), 265-319. 
[Hi-Ya2] A. Hida and N. Yagita, Representation of the double Burnside algebra and cohomology of extraspecial p-group II. J. Algebra 451 (2016), 461493.

[Hu] J. Huebuschmann. Chernclasses for metacyclic groups. Arch. Math. 61 (1993), 124-136.

[Le1] I. J. Leary, The integral cohomology rings of some $p$-groups, Math. Proc. Ca. Phil. Soc. 110 (1991), 25-32.

[Le2] I. J. Leary, The mod- $p$ cohomology rings of some $p$-groups, Math. Proc. Camb. Phil. Soc. 112 (1992), 63-75.

[Lw] G. Lewis, The integral cohomology rings of groups of order $p^{3}$, Trans. Amer. Math. Soc. 132 (1968), 501-529.

[Ma-Pr] J. Martino and S. Priddy, The complete stable splitting for the classifying space of a finite group, Topology 31 (1992), 143-156.

[Mi-Pr] S. Mitchell and S. Priddy, Stable splitting derived from the Steiberg module. Topology 22 (1983), 285-298.

[Ni] G. Nishida, Stable homotopy type of classifying spaces of finite groups. Algebraic and Topological theories ; to the memopry of Dr. Takehiko Miyata. (1985) 391-404.

[Qu] D. Quillen, The spectrum of an equivariant cohomology ring: I, Ann. of Math. 94 (1971), 549-572.

[Ru-Vi] A. Ruiz and A. Viruel, The classification of $p$-local finite groups over the extraspecial group of order $p^{3}$ and exponent $p$, Math. Z. 248 (2004), $45-65$. .

[Te-Ya] M. Tezuka and N. Yagita, On odd prime components of cohomologies of sporadic simple groups and the rings of universal stable elements, J. Algebra 183 (1996), 483-513.

[To1] B. Totaro. The Chow ring of classifying spaces. Proc.of Symposia in Pure Math. "Algebraic K-theory" (1997:University of Washington,Seattle) 67 (1999), 248-281.

[To2] B. Totaro. Group cohomology and algebraic cycles. Cambridge tracts in Math. 204 (2014).

[Th] C.B.Thomas. Characteristic classes and 2-modular representations for some sporadic groups. Lecture note in Math. Vol. 1474 (1990), 371-381.

[Ya1] N. Yagita. Cohomology for groups of $\operatorname{rank}_{p} G=2$ and Brown-Peterson cohomology J. Math. Soc. Japan. 45 (1993) 627-644.

[Ya2] N. Yagita, Stable splitting and cohomology of $p$-local finite groups over the extraspecial $p$-group of order $p^{3}$ and exponent $p$, Geometry and Topology Monographs 11 (2007), 399-434.

[Ya3] N. Yagita. Chow rings of nonabelian $p$-groups of order $p^{3}$. J. Math. Soc. Japan. 64 (2012) 507-531.

[A. Hida] Department of Mathematics, Faculty of Education, Saitama UniVERSity, SAITAMA, JAPAN

E-mail address: ahida@mail.saitama-u.ac.jp

[N. Yagita] Department of Mathematics, Faculty of Education, Ibaraki University, Mito, Ibaraki, Japan

E-mail address: yagita@mx.ibaraki.ac.jp 